\title{
Three-family particle physics models from global F-theory compactifications
}

\author{
Mirjam Cvetič, ${ }^{a}$ Denis Klevers, ${ }^{b}$ Damián Kaloni Mayorga Peña, ${ }^{c}$ \\ Paul-Konstantin Oehlmann ${ }^{d}$ and Jonas Reuter ${ }^{d}$ \\ ${ }^{a}$ Department of Physics and Astronomy, University of Pennsylvania, \\ Philadelphia, PA 19104-6396, U.S.A. \\ ${ }^{b}$ Theory Group, Physics Department, CERN, \\ CH-1211, Geneva 23, Switzerland \\ ${ }^{c}$ The Abdus Salam International Centre for Theoretical Physics, \\ Strada Costiera 11, 34151, Trieste, Italy \\ ${ }^{d}$ Bethe Center for Theoretical Physics, Physikalisches Institut der Universität Bonn, \\ Nussallee 12, 53115 Bonn, Germany \\ E-mail: cvetic@hep.upenn.edu, Denis.Klevers@cern.ch, dmayorga@ictp.it, \\ oehlmann@th.physik.uni-bonn.de, jreuter@th.physik.uni-bonn.de
}

ABSTRACT: We construct four-dimensional, globally consistent F-theory models with three chiral generations, whose gauge group and matter representations coincide with those of the Minimal Supersymmetric Standard Model, the Pati-Salam Model and the Trinification Model. These models result from compactification on toric hypersurface fibrations $X$ with the choice of base $\mathbb{P}^{3}$. We observe that the F-theory conditions on the $G_{4}$-flux restrict the number of families to be at least three. We comment on the phenomenology of the models, and for Pati-Salam and Trinification models discuss the Higgsing to the Standard Model. A central point of this work is the construction of globally consistent $G_{4}$-flux. For this purpose we compute the vertical cohomology $H_{V}^{(2,2)}(X)$ in each case and solve the conditions imposed by matching the M- and F-theoretical 3D Chern-Simons terms. We explicitly check that the expressions found for the $G_{4}$-flux allow for a cancellation of D3brane tadpoles. We also use the integrality of 3D Chern-Simons terms to ensure that our $G_{4}$-flux solutions are adequately quantized.

KEYwords: Beyond Standard Model, F-Theory, Supersymmetric Standard Model, GUT

ARXiv EPRINT: 1503.02068 


\section{Contents}

1 Introduction 1

2 Tools \& strategies for four-dimensional model building 4

3 Minimal supersymmetric standard model: $G_{F_{11}}=\mathrm{SU}(3) \times \mathrm{SU}(2) \times \mathrm{U}(1) \quad 9$

3.1 The geometry of gauge symmetry and particle representations 9

$3.2 G_{4}$-flux and matter chiralities $\quad 11$

$\begin{array}{lll}3.3 & 4 \mathrm{D} \text { anomaly cancelation and family structure } & 14\end{array}$

$\begin{array}{ll}3.4 & \text { Phenomenological discussion } \\ & 16\end{array}$

4 Pati-Salam model: $G_{F_{13}}=\left(\mathrm{SU}(4) \times \mathrm{SU}(2)^{2}\right) / \mathbb{Z}_{2} \quad 18$

$\begin{array}{ll}\text { 4.1 The geometry of gauge group and matter representations } & 18\end{array}$

$\begin{array}{lll}4.2 G_{4} \text {-flux and chiral generations } & 19\end{array}$

$\begin{array}{lll}4.3 & \text { Phenomenological discussion } & 22\end{array}$

5 Trinification model: $G_{F_{16}}=\left(\mathrm{SU}(3)^{3}\right) / \mathbb{Z}_{3} \quad 26$

5.1 The geometry of gauge group and matter representations 26

$\begin{array}{ll}5.2 G_{4} \text {-flux and chiralities } & 28\end{array}$

$\begin{array}{lll}5.3 & \text { Phenomenological discussion } & 30\end{array}$

6 Conclusions 33

$\begin{array}{ll}\text { A Hodge numbers of CY-fourfolds } & 35\end{array}$

B Concrete toric lattice polyhedra of 4D chiral models 37

\section{Introduction}

The construction of fully fledged particle physics models which reproduce the phenomenology of the Standard Model, while providing generic predictions for its behavior at higher scales remains a very active part of the research in string theory. One active front in this direction is F-theory [1], where the geometrization of certain properties of non-perturbative Type IIB string theory allows for a very systematic understanding and engineering of the gauge symmetry, particle content and the interactions in a given model.

Most model building endeavors in F-theory have appealed to an underlying SU(5) gauge group (for a non exhaustive list of works see [2-16]). This is partly due to the simple group theoretical embedding of the standard model gauge group and its representations into SU(5), with its well earned merit for gauge coupling unification. ${ }^{1}$ In addition this gives

\footnotetext{
${ }^{1}$ For a detailed discussion on gauge coupling unification in SU(5) GUTs see e.g. [17, 18].
} 
the advantage of having the full gauge symmetry concentrated on a single divisor in the base that allows for a local treatment of certain features of the model [2-5]. Nevertheless, the increasing understanding of many global issues has prompted interest in alternative models which aim either at the direct construction of the bare $\mathrm{MSSM}^{2}$ [21-23], as well as alternative grand unification schemes such as the Pati-Salam model or Trinification, among others. These models have the advantage that they do not suffer from the pathological group theoretical puzzles inherent to SU(5), such as the doublet triplet splitting problem. These schemes constitute also a very promising alternative in other corners of the string landscape such as perturbative Type IIA/B and the heterotic string (see e.g. [24-26]).

In the model building program one aims at reproducing certain features of the particle physics models such as appropriate gauge symmetries, particle representations, the right number of generations and, at least, the possibility to generate a hierarchy in the Yukawa textures (for reviews on this topic in an F-theory context see e.g. [27, 28]). As for the gauge symmetries, the appearance of non-Abelian factors has been understood since the beginning of F-theory and can be tracked by the degeneration of the elliptic fiber over codimension one surfaces on the base [29-31], see [32] for recent refinements. Abelian gauge symmetries are due to the presence of sections of the elliptic fibration, in addition to the so-called zero section of the Weierstrass model [30, 33, 34]. Being related to global objects, as well as being essential tools for controlling the phenomenology of particle physics models, $\mathrm{U}(1)$ symmetries have pushed the F-theory model building program towards a more global picture. Similarly, the charged matter representations can be tracked by degenerations of the elliptic curve at codimension two in the base [35], see also [36] for the discussion of higher symmetric representation. In order to achieve a chiral spectrum, the addition of $G_{4}$-flux is necessary, see [37,38] for first global examples. The intersection of matter curves at codimension three leads to the geometrically allowed couplings of the model. In F-theory the hierarchy for the Yukawas is possible since generically, the Yukawas for one family are generated geometrically while for the other two of these couplings arise from instanton or flux contributions that are significantly small.

The compactification spaces which are commonly used for constructing $4 \mathrm{D} \mathcal{N}=1$ effective theories in F-theory are genus one fibered Calabi-Yau (CY) fourfolds. The global model building process is divided into two steps, the first step being the construction of appropriate compactification manifolds exhibiting the desired fiber degenerations which lead to the appropriate gauge symmetry, matter and interactions. The second step is concerned with the construction of appropriate $G_{4}$-flux to account for the desired chirality in the spectrum.

Regarding the construction of suitable CY-fourfolds, the efforts have been divided in two fronts: the first pushes towards the classification and construction of all admissible bases [39-41], see also [42-45] for the related study of non-compact bases. The second is more concerned with the construction of genus-one or elliptic fibers $\mathcal{C}$ which naturally allow for certain generic features of the compactification. In this direction, there have been two

\footnotetext{
${ }^{2}$ See $[19,20]$ for earlier attempts to get the standard model gauge group from a deformation of the SU(5) singularity.
} 
major conceptual extensions: one is related to the construction of elliptic curves exhibiting an ever growing number of rational points [13-15, 46-62]. These permit the construction of elliptically fibered CY-manifolds with a certain number of rational sections and hence, a non trivial Mordell-Weil (MW) group of rational sections. While the free part of the MW-group yields the U(1)-gauge fields in F-theory [29], the torsion part is responsible for the presence of non-simply connected gauge groups [33] and its effects are seen at codimension two as it forbids certain representations to be part of the theory. The other conceptual extensions has to do with fibers which entirely lack rational points. These lead to genus-one fibrations which do not have any section [63-65]. Nevertheless, this type of fibrations is suitable for F-theory as their associated Jacobian fibration does have a section and describes the same physics. In the genus-one fibrations, the presence of an $m$-sections has been unveiled as the geometric object responsible for the presence of discrete gauge symmetries in their associated effective SUGRA theory [22, 66-69].

There is a natural framework which provides the simplest examples for the two kinds of fibers described above: the 16 inequivalent 2D toric varieties [70, 71]. By describing the genus-one fiber as an algebraic curve in any of these toric ambient spaces one ends up with any of the possible cases of elliptic fibers with up to four independent rational points as well as genus-one curves with two- and three-sections. In [22], a systematic analysis of the effective six dimensional theories stemming from compactification of F-theory on any of these 16 toric hypersurface fibrations over an arbitrary complex-two dimensional base was done. Abandoning the paradigm of the holomorphic zero section and allowing it to be simply rational, all resolution divisors inherited from the corresponding ambient space descend to Cartan divisors on the corresponding CY-manifold. In this fashion, it was possible to deduce the intrinsic gauge symmetry of each toric hypersurface fibration, without the necessity to introduce further specializations of the geometry such as tops [7274], which appear in algorithmic approaches to F-theory model building [58]. Among these intrinsic gauge symmetries, groups which are familiar for particle physicists such as $\mathrm{SU}(3) \times \mathrm{SU}(2) \times \mathrm{U}(1), \mathrm{SU}(4) \times \mathrm{SU}(2)^{2} / \mathbb{Z}_{2}$ and $\mathrm{SU}(3)^{3} / \mathbb{Z}_{3}$ appear naturally. Even more interestingly, the matter representations that are possible in each of these models coincide with those needed for the MSSM, Pati-Salam model and Trinification, respectively. While the analysis of [22] was made in six dimensions, these findings carry over to $4 \mathrm{D}$ as well. Another important feature of the six-dimensional models is the existence of a so called "toric Higgsing" (or a chain of those) which allows for a pictorial, toric interpretation of Higgsings from the Pati-Salam and Trinification models down to the MSSM.

In this work we study the four dimensional-version of the MSSM, the Pati-Salam model and Trinification engineered by F-theory on toric hypersurface fibrations $X$. For the first time we construct explicit, globally consistent, three-family models with the chiral matter content of the MSSM, Pati-Salam model and Trinification. These models result from three different toric hypersurface fibrations over a fixed base $B=\mathbb{P}^{3}$. We construct the vertical cohomology $H_{V}^{(2,2)}(X)$ and provide the most general expression for the $G_{4}$-flux in each case. These expressions are shown to comply with all conditions on M-theory Chern-Simons terms imposed by duality with F-theory and allow for a D3-brane tadpole canceling solution which involves an integral and positive number of D3-branes. Since 
the determination of an integral basis for the vertical cohomology cohomology $H_{V}^{(2,2)}(X)$, $G_{4}$-flux quantization is checked indirectly by ensuring quantization of the induced 3D Chern-Simons terms. Regarding the phenomenology of the considered models we have two main drawbacks: since we have no control over the vector-like sector of the theory, it is not guaranteed that we have the vector-like pairs needed for the breaking of electroweak symmetry neither for the breaking of the Pati-Salam or Trinification groups down to the Standard Model gauge group. In addition, we have only stated the trilinear couplings which are generated geometrically and have argued that under the assumption of the presence of a light pair of Higgses, these would allow for the generation of a hierarchy. However, the currently applicable tools do not allow us to provide to perform a quantitative analysis.

This paper is organized as follows: in section 2 we summarize the general procedure to construct 4D chiral models from F-theory and shortly review the tools needed for our analysis: the general features of elliptic fibrations, $G_{4}$-flux and $G_{4}$-flux consistency conditions in F-theory. In section 3 we discuss the elliptic fibration leading to the gauge group and matter content of the MSSM. There, we also compute $G_{4}$-flux and the resulting 4D matter chiralities. Similar as in the standard model, we see that the exact cancelation of anomalies (without Green Schwarz counterterms for anomalies involving $\mathrm{U}(1)_{Y}$ ) enforces a family structure. In this regime we scan over all allowed strata in the moduli space of the CY-manifold $X_{F_{11}}$ with base $\mathbb{P}^{3}$ and compute the smallest number of families for which the D3 brane tadpole is canceled with a positive integral number of D3-branes. We observe that three is in fact the smallest permitted number of families in this model. The same observation holds also for Pati-Salam and Trinification models. In addition we give closed formulas for the Hodge structure of all fibrations for the choice of base $B=\mathbb{P}^{3}$ in appendix A. We generically find $h^{(2,1)}=0$ which restricts cosmological applications. We conclude this section with a brief discussion of the phenomenology of the model under the assumption that a light pair of Higgs fields is present. In sections 4 and 5 we present a similar discussion of the Pati-Salam and Trinification models as in section 3. In addition, we comment on the Higgsing down to the Standard Model gauge group. We indeed find that there exist three-family models both for Pati-Salam and Trinification, that Higgs down to three-family Standard models. Finally in section 6 we present our conclusions and discuss future possible directions of research. Appendix A contains a brief account on the computation of Hodge numbers of CY-fourfolds given as toric hypersurfaces and applications to the considered cases $X_{F_{11}}, X_{F_{13}}$ and $X_{F_{16}}$ with base $B=\mathbb{P}^{3}$. Appendix B contains the explicit lattice polytopes for the five-dimensional toric ambient spaces of all considered toric hypersurface fibrations.

\section{Tools \& strategies for four-dimensional model building}

In this section, which serves as a preparation for the analysis in sections 3,4 and 5, we outline the basic techniques for building F-theoretic models of particle physics. Although many of the presented methods are applicable to general Calabi-Yau (CY) fourfolds $X$, we focus here on the case of the toric hypersurface fibrations as studied in [22]. Except for the discussion of $G_{4}$-flux quantization, this section is mainly a concise review on the 
construction of chiral 4D F-theory models, following closely [15] to which we refer for further details. In an accompanying appendix A we discuss the computation of Hodge numbers of CY-fourfolds $X$ given as toric hypersurfaces. The reader interested in the phenomenological results can safely skip this section and continue with section 3 .

F-theory geometry: on the geometry side, the starting point for the construction of an F-theory model is the choice of a three-dimensional base manifold $B$ as well as the genus-one or elliptic fiber of the Calabi-Yau fourfold $X$. As a next step all codimension one, two and three singularities of the fibration have to be analyzed in order to determine the gauge group $G$, matter content, i.e. the representations and their matter curves in $X$, and the Yukawa couplings of the 4D effective theory of F-theory.

In order to being able to construct $G_{4}$-flux we have to compute the cohomology ring of $X$. For a CY-fourfold given as a toric hypersurface (or complete intersection) fibration, which is the case of interest of this work, the full cohomology ring of $X$ as a quotient polynomial ring generated by $H^{1,1}(X)[15,75-77]$. Concretely, for a CY-fourfold $X$ with a given toric base $B$, we choose a basis $D_{A}, A=0, \ldots, h^{(1,1)}(X)-1$, for the divisor group $H^{1,1}(X)$. Then the cohomology ring of $X$ is given by the polynomial ring in the $D_{A}$ divided by the Stanley-Reisner (SR) ideal of the ambient toric variety ${ }^{3}$ of $X$. The reduction by the Stanley-Reisner ideal works in our case as we have no non-toric divisors. There can be nontoric divisors at corners of the allowed region for $\mathcal{S}_{7}$ and $\mathcal{S}_{9}$ for the base $\mathbb{P}^{3}$, cf. the discussion in [15]. As our focus is on chirality inducing $G_{4}$-flux, we are primarily interested in the subgroup $H_{\mathrm{V}}^{2,2}(X)$ of the fourth cohomology of $X$, the primary vertical cohomology [78]. It is given by the quotient ring at grade two, that is constructed by forming all possible products $D_{A} \cdot D_{B}$. These are linearly dependent. Thus, we compute the rank of the inner product matrix on these elements, which yields the dimension $h_{V}^{(2,2)}(X)$, and choose an appropriate basis. The topological metric on this basis is denoted by $\eta^{(2)}$.

We emphasize that the full Chern class of the CY-manifold $X$ can be computed independently of the base $B$ by using the adjunction formula and the total Chern class of the ambient space, see [15] for more details. Of particular interest for F-theory are the second Chern class $c_{2}(X)$ and the Euler number $\chi(X)$ of $X$.

Constraints on $\boldsymbol{G}_{\mathbf{4}}$-flux in F-theory: $\quad$ F-theory on $X \times S^{1}$ and M-theory on $X$ are dual to each other [79]. Thus, consistent $G_{4}$-flux in a four-dimensional F-theory compactification on $X$ is understood as $G_{4}$-flux in the dual M-theory compactification on the same $X$ to three dimensions, so that the $G_{4}$-flux obeys additional consistency conditions. These consistency conditions follow from requiring that the three-dimensional effective actions of $\mathrm{F}$ - and $\mathrm{M}$ theory agree, which can be used to derive the full effective action of F-theory [80].

In M-theory, $G_{4}$-flux has to fulfill two basic conditions. First, it must obey the following quantization condition [81]:

$$
G_{4}+\frac{c_{2}(X)}{2} \in H^{4}(X, \mathbb{Z})
$$

\footnotetext{
${ }^{3}$ The reduction by the Stanley-Reisner ideal works in our case as we have no non-toric divisors. There can be non-toric divisors at corners of the allowed region for $\mathcal{S}_{7}$ and $\mathcal{S}_{9}$ for the base $\mathbb{P}^{3}$, cf. the discussion in $[15]$.
} 
Second, the cancelation of M2-brane tadpoles, which lift to D3-brane tadpoles in Type IIB strings and F-theory, requires the equality [82, 83]

$$
\frac{\chi(X)}{24}=n_{\mathrm{D} 3}+\frac{1}{2} \int_{X} G_{4} \wedge G_{4}
$$

where $n_{\mathrm{D} 3}$ denotes the number of D3-branes. As mentioned before, we will focus here on special $G_{4}$-flux that is entirely in the subgroup $H_{V}^{(2,2)}(X) .^{4}$

For compatibility with the duality between M- and F-theory, we need to impose additional conditions on the $G_{4}$-flux. These are most easily formulated in terms of conditions on the Chern-Simons (CS) terms for the three-dimensional vectors on the Coulomb branch of the effective action of the M-theory compactification on the CY-fourfold $X$. On the M-theory side, these CS-terms are given by [87]

$$
\Theta_{A B}^{\mathrm{M}}=\int_{X} G_{4} \wedge D_{A} \wedge D_{B}
$$

where here and in the following, Poincaré duality is always understood. We note that the 3D CS-terms have to obey the quantization condition $\Theta_{A B}^{M} \in \mathbb{Z}$ or $\mathbb{Z} / 2$, see e.g. [88, 89] for recent discussions. We note that these quantization conditions are expected to be equivalent to the $G_{4}$-flux quantization conditions (2.1) [85].

In the dual F-theory side the same CS-terms, denoted now by $\Theta_{A B}^{\mathrm{F}}$, have two contributions. First, we can have classical CS-terms $\Theta_{\mathrm{cl}, A B}^{F}$, which either descend from 4D to 3D from gaugings of axions or which correspond to circle fluxes [90]. Second, CS-terms on the 3 D Coulomb branch receive one-loop corrections from integrating out massive fermions [9193]. In the duality between M- and F-theory, it is crucial to include all Kaluza-Klein (KK) states in the loop $[15,54],{ }^{5}$ yielding the full loop corrected CS-terms expression

$$
\Theta_{A B}^{F}=\Theta_{\mathrm{cl}, A B}^{F}+\frac{1}{2} \sum_{\underline{q}} n(\underline{q}) q_{A} q_{B} \operatorname{sign}\left(q_{A} \zeta^{A}\right) .
$$

Here $n(q)$ is the number of $3 \mathrm{D}$ fermions with charge vector $q=\left(q_{0}, q_{\alpha}, q_{i}, q_{m}\right)$. It includes the charge $q_{0}$ w.r.t. the $3 \mathrm{D}$ graviphoton, i.e. the KK-level of states, the charges $q_{\alpha}, \alpha=$ $1, \ldots, h^{(1,1)}(B)$, under $3 \mathrm{D}$ vectors dual to the Kähler moduli of $B$, the charges $q_{i}, i=$ $1, \ldots, \operatorname{rk}(G)$, and $q_{m}, m=1, \ldots, r$, w.r.t. to $4 \mathrm{D}$ Cartan gauge fields of the non-Abelian gauge group $G$ of F-theory and the $r \mathrm{U}(1)$ gauge fields, respectively. The real parameters $\zeta^{A}$ are the Coulomb branch parameters.

Duality requires an identification of the CS-terms on the F-theory side with those in (2.3) on the M-theory side [14, 15, 51, 54, 94, 95],

$$
\Theta_{A B} \equiv \Theta_{A B}^{M} \stackrel{!}{=} \Theta_{A B}^{F}
$$

This immediately leads to additional restrictions on the CS-terms in F-theory [15, 37, 51, 54], because certain CS-terms $\Theta_{A B}^{F}$ in F-theory computed according to (2.4) are identically

\footnotetext{
${ }^{4}$ For recent analysis of horizontal $G_{4}$-flux in F-theory, see [77, 84-86].

${ }^{5}$ See also [57] for the case of CS-terms in 5D M-/F-theory duality.
} 
zero. Physically, the implied constraints on the $G_{4}$-flux ensure the absence of circle flux in the circle compactification from F- to M-theory, an unbroken non-Abelian gauge group in $4 \mathrm{D}$ due to the absence of axion gaugings and the absence of non-geometric effects,

$$
\Theta_{0 \alpha}=\Theta_{i \alpha}=\Theta_{\alpha \beta}=0
$$

Here we have to chose the basis $D_{A}$ of $H^{(1,1)}(X)$ so that index 0 corresponds to the zero section $\hat{s}_{0}$ of the fibration of $X, \alpha=1, \ldots, h^{(1,1,)}(B)$, labels the vertical divisors induced from the base $B, i=1, \ldots, \operatorname{rk}(G)$ labels the Cartan divisors of $X$, where $G$ as before is non-Abelian part of the F-theory gauge group, and $m=1, \ldots, r$ labels the $r \mathrm{U}(1)$-factors corresponding to Shioda maps $\sigma\left(\hat{s}_{m}\right)$ of the rank $r$ Mordell-Weil (MW) group of rational sections $\hat{s}_{m}$ of $X$.

Chiralities in F-theory and $\boldsymbol{G}_{\mathbf{4}}$-flux quantization: in order to calculate the matter chiralities $\chi(\mathbf{R})$ for a given matter representation $\mathbf{R}$ in a four-dimensional F-theory compactification, we need to integrate the $G_{4}$-flux over a corresponding matter surface in $X$. The relevant matter surface $\mathcal{C}_{\mathbf{R}}^{w}$ is given as the rational surface constructed by fibering a $\mathbb{P}^{1}$ carrying the weight $\mathbf{w}$ of the representation $\mathbf{R}$ over the corresponding matter curve in the base $B$. The $4 \mathrm{D}$ chirality of $\mathbf{R}$ is computed as

$$
\chi(\mathbf{R})=n(\mathbf{R})-n(\overline{\mathbf{R}})=\int_{\mathcal{C}_{\mathbf{R}}^{w}} G_{4},
$$

where $n(\mathbf{R})$ denotes the number of left-chiral Weyl fermions in the representation $\mathbf{R}$.

Technically, the determination of the $\mathcal{C}_{\mathbf{R}}^{w}$ can be involved and requires the computation of the homology class of prime ideals describing the given matter surface. This can be done using the resultant technique that was applied first in $[15,56]$ for F-theory and will be exemplify for the three examples studied in this work. As a consistency check of our geometric computations, following $[15,51,54]$, we use the matching condition (2.5) of the CS-terms to double-check the $4 \mathrm{D}$ chiralities calculated using (2.7).

Finally, let us comment on $G_{4}$-flux quantization. In principal, in order to address $G_{4}$-flux quantization we have to expand $G_{4}$ and $c_{2}(X)$ in an integral basis for $H_{V}^{(2,2)}(X)$ and check the condition (2.1). This integral basis can be determined employing mirror symmetry techniques $[77,84,86]$. Since this is beyond the scope of this work, we will apply an indirect approach to ensure integral $G_{4}$-flux.

Here we exploit that $G_{4}$-flux quantization (2.1), the integrality of the number $n_{\mathrm{D} 3}$ of D3-branes, that is a necessary condition for quantized $G_{4}$-flux [81], the integrality of the CS-terms (2.3) and of the chiralities (2.7) are obviously linked to each other. Thus, our strategy will be the following. First, we compute all chiralities $\chi(\mathbf{R})$ using $(2.7)$. Then, we parametrize the coefficients in the expansion of the $G_{4}$-flux w.r.t. a basis of $H_{V}^{(2,2)}(X)$ in terms of these integral chiralities. We then impose the necessary condition of integrality and positivity of $n_{\mathrm{D} 3}$. This will yield in turn constraints in form of lower bounds on the $4 \mathrm{D}$ chiralities. Next, we impose, if possible, a family structure on our model. Finally, we check that for this phenomenologically preferred choice of $G_{4}$-flux all CS-terms are integral, which ensures that the quantization condition (2.1) is obeyed. We remark that there might exist 
a finer basis non-diagonal in vertical and horizontal cohomology for which more integral $n_{D 3}$ chiral family solutions exist. In contrast we only focus on the vertical part, that might be a multiple of this minimal component, such that it is integral on its own.

Toric hypersurface fibrations for 4D chiral F-theory models: in order to introduce some notation used throughout this work, we conclude this introductory section with a very brief review of CY-fourfolds $X$ constructed as toric hypersurface fibrations. A detailed account on this subject can be found in [22].

We consider here elliptically fibered Calabi-Yau manifolds $X_{F_{i}}$ whose elliptic fiber is realized as the general CY-hypersurface in a $2 \mathrm{D}$ toric variety $\mathbb{P}_{F_{i}}$ associated to one of the $2 \mathrm{D}$ reflexive polyhedra $F_{i}$. Here we focus on the polyhedra $F_{11}, F_{13}$ and $F_{16}$ in [22], that naturally yield phenomenologically interesting models. In these cases, the corresponding toric ambient varieties $\mathbb{P}_{F_{i}}$ of the elliptic fiber are blow-ups of $\mathbb{P}^{2}$. The elliptic curves in all considered cases are consequently given as an appropriate specialization of the general cubic

$$
p=s_{1} u^{3}+s_{2} u^{2} v+s_{3} u v^{2}+s_{4} v^{3}+s_{5} u^{2} w+s_{6} u v w+s_{7} v^{2} w+s_{8} u w^{2}+s_{9} v w^{2}+s_{10} w^{3} .
$$

Here the coefficients $s_{i}$ take values in a field $K$ and $[u: v: w]$ are projective coordinates on $\mathbb{P}^{2}$.

An elliptic fibration $X_{F_{i}}$ with fiber given by (2.8) or specializations thereof is constructed by first fibering the toric ambient space $\mathbb{P}_{F_{i}}$ over a chosen base $B$, then imposing (2.8) and finally demanding the CY-condition. In this procedure, the coordinates $[u: v: w]$ and the coefficients $s_{i}$ in (2.8) are lifted to sections of appropriate line bundles on $B$. The $\mathrm{CY}$-condition fixes these line bundles to the following:

\begin{tabular}{|c|c|}
\hline section & Line Bundle \\
\hline$u$ & $\mathcal{O}\left(H+\mathcal{S}_{9}+\left[K_{B}\right]\right)$ \\
$v$ & $\mathcal{O}\left(H+\mathcal{S}_{9}-\mathcal{S}_{7}\right)$ \\
$w$ & $\mathcal{O}(H)$ \\
\hline
\end{tabular}

\begin{tabular}{|c|c|}
\hline section & Line Bundle \\
\hline$s_{1}$ & $\mathcal{O}_{B}\left(3\left[K_{B}^{-1}\right]-\mathcal{S}_{7}-\mathcal{S}_{9}\right)$ \\
$s_{2}$ & $\mathcal{O}_{B}\left(2\left[K_{B}^{-1}\right]-\mathcal{S}_{9}\right)$ \\
$s_{3}$ & $\mathcal{O}_{B}\left(\left[K_{B}^{-1}\right]+\mathcal{S}_{7}-\mathcal{S}_{9}\right)$ \\
$s_{4}$ & $\mathcal{O}_{B}\left(2 \mathcal{S}_{7}-\mathcal{S}_{9}\right)$ \\
$s_{5}$ & $\mathcal{O}_{B}\left(2\left[K_{B}^{-1}\right]-\mathcal{S}_{7}\right)$ \\
$s_{6}$ & $K_{B}^{-1}$ \\
$s_{7}$ & $\mathcal{O}_{B}\left(\mathcal{S}_{7}\right)$ \\
$s_{8}$ & $\mathcal{O}_{B}\left(\left[K_{B}^{-1}\right]+\mathcal{S}_{9}-\mathcal{S}_{7}\right)$ \\
$s_{9}$ & $\mathcal{O}_{B}\left(\mathcal{S}_{9}\right)$ \\
$s_{10}$ & $\mathcal{O}_{B}\left(2 \mathcal{S}_{9}-\mathcal{S}_{7}\right)$ \\
\hline
\end{tabular}

Here, $\mathcal{O}(D)$ denotes the line bundle associated to a divisor $D,{ }^{6} H$ is the hyperplane on $\mathbb{P}^{2},\left[K_{B}^{-1}\right]$ is the anti-canonical divisor of $B$ and $\mathcal{S}_{7}, \mathcal{S}_{9}$ are the divisor classes of $s_{7}, s_{9}$, respectively. We note that the table on the right hand side in (2.9) applies for all examples studied below.

\footnotetext{
${ }^{6} \mathrm{~A}$ subscript indicates the space over which this line bundle is defined, e.g. $\mathcal{O}_{B}(D)$ denotes a line bundle over $B$. If a subscript is omitted, the line bundle lives on the ambient space of $X$.
} 

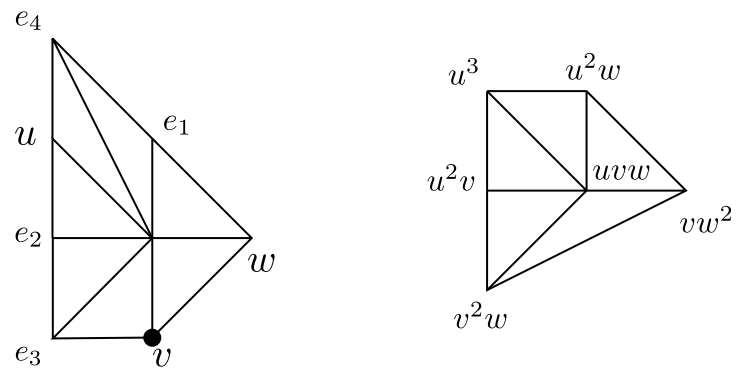

\begin{tabular}{|c|c|}
\hline section & Line Bundle \\
\hline$u$ & $\mathcal{O}\left(H-E_{1}-E_{2}-E_{4}+\mathcal{S}_{9}+\left[K_{B}\right]\right)$ \\
\hline$v$ & $\mathcal{O}\left(H-E_{2}-E_{3}+\mathcal{S}_{9}-\mathcal{S}_{7}\right)$ \\
\hline$w$ & $\mathcal{O}\left(H-E_{1}\right)$ \\
\hline$e_{1}$ & $\mathcal{O}\left(E_{1}-E_{4}\right)$ \\
\hline$e_{2}$ & $\mathcal{O}\left(E_{2}-E_{3}\right)$ \\
\hline$e_{3}$ & $\mathcal{O}\left(E_{3}\right)$ \\
\hline$e_{4}$ & $\mathcal{O}\left(E_{4}\right)$ \\
\hline
\end{tabular}

Figure 1. The toric diagram of polyhedron $F_{11}$ and its dual. The zero section is indicated by the dot. In the accompanying table we indicate the divisor classes of the fiber coordinates.

\section{Minimal supersymmetric standard model: $G_{F_{11}}=\mathrm{SU}(3) \times \mathrm{SU}(2) \times$ $\mathrm{U}(1)$}

In this section we discuss an F-theory compactification on the elliptically fibered CYmanifold $X_{F_{11}}$ which yields precisely the gauge group and representation content of the Minimal Supersymmetric Standard Model (MSSM) [22].

In section 3.1 we elaborate on the basic geometrical properties of $X_{F_{11}}$ that encode the gauge symmetry, including the $\mathrm{U}(1)$ generator, as well as the matter representations. While these observations are model independent, we further specialize to the simple base $B=\mathbb{P}^{3}$. For this specific case we compute the vertical cohomology $H_{V}^{(2,2)}\left(X_{F_{11}}\right)$ in section 3.2. Using these results, we explicitly construct $G_{4}$-flux consistent with all F-theory consistency constraints. We compute the induced $4 \mathrm{D}$ chiralities of the matter representations, that we double-check employing 3D CS-terms and M-/F-theory duality. Next in section 3.3 we discuss $4 \mathrm{D}$ anomaly cancelation and the properties of models which exhibit a complete family structure, in particular the existence of three family models with positive and integral D3-brane charge and quantized $G_{4}$-flux. In section 3.4 we conclude with some comments on the phenomenology of the three family models we found.

The elliptic fibration $X_{F_{11}}$ has been completely analyzed in [22], to which we refer for more details on its codimension one, two and three singularities and the corresponding $6 \mathrm{D}$ F-theory compactification. The relevant results are summarized in section 3.1. The reader less interested in the technical details can directly jump to the $4 \mathrm{D}$ chiralities in (3.17) and the following discussions.

\subsection{The geometry of gauge symmetry and particle representations}

The elliptic fiber which is used to engineer F-theory models that naturally exhibit the gauge symmetry of the standard model is given as the CY-hypersurface

$$
\begin{aligned}
p_{F_{11}}= & s_{1} e_{1}^{2} e_{2}^{2} e_{3} e_{4}^{4} u^{3}+s_{2} e_{1} e_{2}^{2} e_{3}^{2} e_{4}^{2} u^{2} v+s_{3} e_{2}^{2} e_{3}^{2} u v^{2}+s_{5} e_{1}^{2} e_{2} e_{4}^{3} u^{2} w \\
& +s_{6} e_{1} e_{2} e_{3} e_{4} u v w+s_{9} e_{1} v w^{2}
\end{aligned}
$$

in the toric ambient space $\mathbb{P}_{F_{11}}$. Its toric data is summarized in figure 1. The divisor classes in $\mathbb{P}_{F_{11}}$ are $H$, the hyperplane class of $\mathbb{P}^{2}$, as well as the four exceptional divisors $E_{1}, E_{2}$, $E_{3}$ and $E_{4}$. 


\begin{tabular}{|c|c|}
\hline Representation & Locus \\
\hline$(\mathbf{3}, \mathbf{2})_{1 / 6}$ & $V\left(I_{(1)}\right):=\left\{s_{3}=s_{9}=0\right\}$ \\
\hline$(\mathbf{1}, \mathbf{2})_{-1 / 2}$ & $V\left(I_{(2)}\right):=\left\{s_{3}=s_{2} s_{5}^{2}+s_{1}\left(s_{1} s_{9}-s_{5} s_{6}\right)=0\right\}$ \\
\hline$(\overline{\mathbf{3}}, \mathbf{1})_{-2 / 3}$ & $V\left(I_{(3)}\right):=\left\{s_{5}=s_{9}=0\right\}$ \\
\hline$(\overline{\mathbf{3}}, \mathbf{1})_{1 / 3}$ & $V\left(I_{(4)}\right):=\left\{s_{9}=s_{3} s_{5}^{2}+s_{6}\left(s_{1} s_{6}-s_{2} s_{5}\right)=0\right\}$ \\
\hline$(\mathbf{1}, \mathbf{1})_{1}$ & $V\left(I_{(5)}\right):=\left\{s_{1}=s_{5}=0\right\}$ \\
\hline
\end{tabular}

Table 1. Charged matter representations under $\mathrm{SU}(3) \times \mathrm{SU}(2) \times \mathrm{U}(1)$ and corresponding codimension two loci in $X_{F_{11}}$. The charge under the $\mathrm{U}(1)_{Y}$ generator is indicated by a subscript.

Next, an elliptically fibered CY-fourfold $X_{F_{11}}$ with the elliptic fiber (3.1) is constructed by promoting the coefficients $s_{i}$ in the CY-equation to sections of the line bundles of $B$ given in (2.9). The elliptic fibration of $X_{F_{11}}$ is equipped with two independent rational sections

$$
\begin{array}{ll}
\hat{s}_{0}=X_{F_{11}} \cap\{v=0\}: & {\left[1: 0: s_{1}: 1: 1:-s_{5}: 1\right],} \\
\hat{s}_{1}=X_{F_{11}} \cap\left\{e_{4}=0\right\}: & {\left[s_{9}: 1: 1:-s_{3}: 1: 1: 0\right],}
\end{array}
$$

where we have chosen $\hat{s}_{0}$ as the zero section. By computation of the discriminant of the fibration (3.1), one can check that over the loci $\mathcal{S}_{\mathrm{SU}(2)}=\left\{s_{3}=0\right\}$ and $\mathcal{S}_{\mathrm{SU}(3)}=\left\{s_{9}=0\right\}$ the fiber degenerates to $I_{2}$ - and $I_{3}$-fibers giving rise to $\mathrm{SU}(2)$ and $\mathrm{SU}(3)$ gauge symmetries, respectively. The Cartan divisors of these gauge groups are

$$
D_{1}^{\mathrm{SU}(2)}=\left[e_{1}\right], \quad D_{1}^{\mathrm{SU}(3)}=\left[e_{2}\right] \quad D_{2}^{\mathrm{SU}(3)}=[u] .
$$

Having these divisors at hand, one can show that the generator of the U(1) symmetry, that is the Shioda map of $\hat{s}_{1}$, is given by

$$
\sigma\left(\hat{s}_{1}\right)=S_{1}-\tilde{S}_{0}+\left[K_{B}\right]+\frac{1}{2} D_{1}^{\mathrm{SU}(2)}+\frac{1}{3}\left(D_{1}^{\mathrm{SU}(3)}+2 D_{2}^{\mathrm{SU}(3)}\right) .
$$

Here, $S_{1}$ denotes the class of $\hat{s}_{1}$ and we used $\tilde{S}_{0}=S_{0}+\frac{1}{2}\left[K_{B}^{-1}\right]$ [96], where $S_{0}$ is the class of $\hat{s}_{0}$ and $K_{B}^{-1}$ denotes the anti-canonical bundle of the base $B$. The corresponding Néron-Tate height pairing reads

$$
b_{11}=\frac{3}{2}\left[K_{B}^{-1}\right]-\frac{1}{2} \mathcal{S}_{7}-\frac{1}{6} \mathcal{S}_{9} .
$$

Furthermore, there are codimension two singularities in the elliptic fibration $X_{F_{11}}$ which support all matter representations of the Standard Model $^{7}$ as one can see in table 1.

We note that the second Chern class $c_{2}$ and the Euler number of $X_{F_{11}}$ can be computed base independently [61]. They are needed to check the $G_{4}$-flux quantization condition (2.1)

\footnotetext{
${ }^{7}$ At this stage, over the different codimension two loci, matter comes in vector-like pairs. It is the $G_{4}$-flux that induces chiralities for the fields.
} 


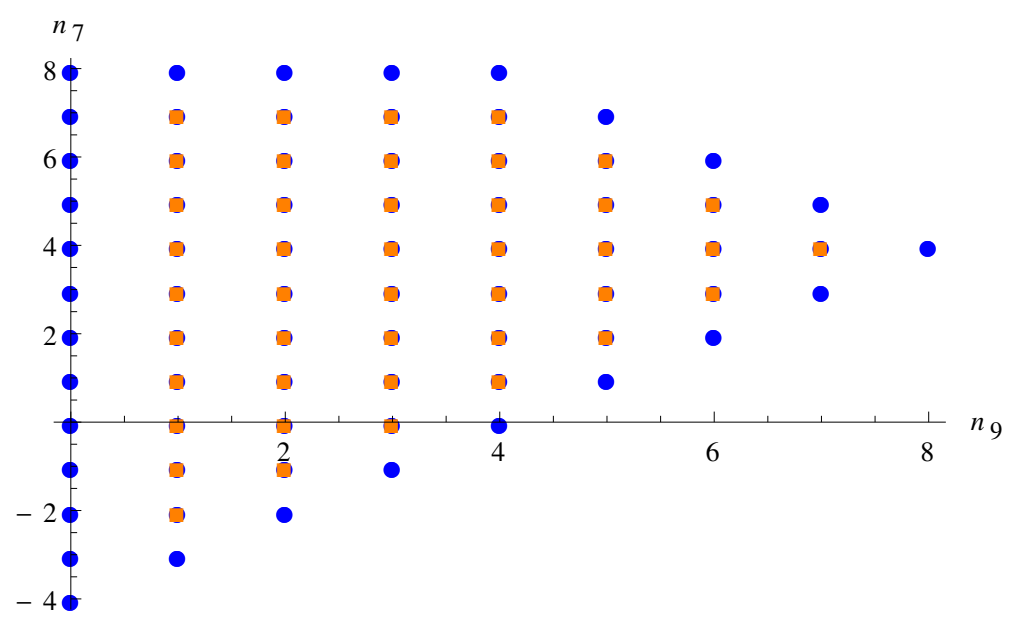

Figure 2. Allowed region for $\left(n_{7}, n_{9}\right)$ for the CY-fourfold $X_{F_{11}}$ with base $\mathbb{P}^{3}$. Orange dots indicate that all SM representations are present and a $G_{4}$-flux admitting $b$ families exists.

as well as the cancelation of D3 tadpoles (2.2). We obtain

$$
\begin{aligned}
c_{2}\left(X_{F_{11}}\right)= & -c_{1}^{2}+c_{2}+c_{1} E_{2}-c_{1} E_{3}-7 c_{1} E_{4}-7 E_{4}^{2}+4 c_{1} H+2 c_{1} \mathcal{S}_{7}+4 E_{1} \mathcal{S}_{7} \\
& +E_{2} \mathcal{S}_{7}+E_{3} \mathcal{S}_{7}+6 E_{4} \mathcal{S}_{7}-4 H \mathcal{S}_{7}-c_{1} \mathcal{S}_{9}-5 E_{1} \mathcal{S}_{9}-3 E_{2} \mathcal{S}_{9} \\
& -E_{3} \mathcal{S}_{9}+3 H \mathcal{S}_{9}-3 \mathcal{S}_{7} \mathcal{S}_{9}+3 \mathcal{S}_{9}^{2} \\
\chi\left(X_{F_{11}}\right)= & 3\left(24 c_{1}^{3}+4 c_{1} c_{2}-16 c_{1}^{2} \mathcal{S}_{7}+8 c_{1} \mathcal{S}_{7}^{2}-18 c_{1}^{2} \mathcal{S}_{9}\right. \\
& \left.+3 c_{1} \mathcal{S}_{7} \mathcal{S}_{9}-3 \mathcal{S}_{7}^{2} \mathcal{S}_{9}+6 c_{1} \mathcal{S}_{9}^{2}+\mathcal{S}_{7} \mathcal{S}_{9}^{2}\right)
\end{aligned}
$$

where $c_{1}$ and $c_{2}$ denote the first and second Chern class of the base $B$, respectively. The divisors $\mathcal{S}_{7}$ and $\mathcal{S}_{9}$ are introduced in (2.9).

For the remainder of this section, we chose the base of the fibration to be $B=\mathbb{P}^{3}$. For this simple choice of base the only vertical divisor is the pullback of the hyperplane class $\mathbb{P}^{3}$, which we denote by $H_{B}$. In this case, we have $c_{1}=4\left[H_{B}\right]$ and $c_{2}=6\left[H_{B}^{2}\right]$ in (3.7). In addition, we readily expand the divisors $\mathcal{S}_{7}, \mathcal{S}_{9}$ in $(2.9)$ needed to specify the fibration $X_{F_{11}}$ in terms of $H_{B}$,

$$
\mathcal{S}_{7}=n_{7} H_{B}, \quad \mathcal{S}_{9}=n_{9} H_{B}, \quad\left[K_{B}^{-1}\right]=4 H_{B},
$$

where $n_{7}$ and $n_{9}$ denote integers. These integers are constrained by requiring effectiveness of all divisor classes in (2.9), that enter the CY-constraint (3.1). This determines a region of allowed values for the pair $\left(n_{7}, n_{9}\right)$, to which we refer to as the allowed region, as depicted in figure 2.

\section{$3.2 G_{4}$-flux and matter chiralities}

For the specific base $B=\mathbb{P}^{3}$, the full SR-ideal of the toric ambient space of $X_{F_{11}}$ is given by ${ }^{8}$

$$
S R_{F_{11}}=\left\{u e_{1}, u w, u v, u e_{3}, e_{4} w, e_{4} v, e_{4} e_{3}, e_{4} e_{2}, e_{1} v, e_{1} e_{3}, e_{1} e_{2}, w e_{3}, w e_{2}, v e_{2}, x_{0} x_{1} x_{2} x_{3}\right\}
$$

\footnotetext{
${ }^{8}$ The SR-ideal of the fiber alone can be found in [22].
} 
where $[u, v, w]$ and the $e_{i}, i=1, \ldots, 4$, are the projective coordinates on the fiber and $x_{j}$ $(j=0,1,2,3)$ are the homogeneous coordinates on the $\mathbb{P}^{3}$ base. A basis for $H^{(1,1)}\left(X_{F_{11}}\right)$ is given by

$$
H^{(1,1)}\left(X_{F_{11}}\right)=\left\langle H_{B}, \tilde{S}_{0}, D_{1}^{\mathrm{SU}(2)}, D_{1}^{\mathrm{SU}(3)}, D_{2}^{\mathrm{SU}(3)}, \sigma\left(\hat{s}_{1}\right)\right\rangle
$$

where we denote, by abuse of notation, divisors and their Poincare dual $(1,1)$-forms by the same symbol.

Next we proceed with the computation of the full vertical cohomology ring of $X_{F_{11}}$ following [15]. To set up its computation as a quotient ring, we need the SR ideal (3.9), the basis of divisors (3.8) as well as the intersection numbers,

$$
H_{B}^{3} \cdot S_{0}^{2}=-1, \quad H_{B}^{3} \cdot S_{1}^{2}=-1,
$$

which follow from the toric intersections in $\mathbb{P}_{F_{11}}$. The quartic intersections of $X_{F_{11}}$ can be readily computed and a canonical basis for $H^{(3,3)}\left(X_{F_{11}}\right)$ is obtained by duality to $H^{(1,1)}\left(X_{F_{11}}\right)$. We obtain generators for $H_{V}^{(2,2)}\left(X_{F_{11}}\right)$ by constructing all possible products of two divisors in $H^{(1,1)}\left(X_{F_{11}}\right)$. We evaluate the rank of the inner product on these generators as

$$
\operatorname{dim} H^{2,2}\left(X_{F_{11}}\right)=7
$$

As a basis for $H_{V}^{(2,2)}\left(X_{F_{11}}\right)$ we choose the seven elements

$$
H_{V}^{(2,2)}\left(X_{F_{11}}\right)=\left\langle\left(H_{B}\right)^{2}, H_{B} \tilde{S}_{0}, D_{1}^{\mathrm{SU}(2)} H_{B}, D_{1}^{\mathrm{SU}(3)} H_{B}, D_{2}^{\mathrm{SU}(3)} H_{B}, H_{B} \sigma\left(\hat{s}_{1}\right), \tilde{S}_{0}^{2}\right\rangle .
$$

We can then expand the $G_{4}$-flux in terms of the this basis. Imposing the conditions (2.6) required by a match of M- and F-theory CS-terms leads to five conditions on the $G_{4}$-flux, yielding the following two parameter $G_{4}$-flux on $X_{F_{11}}$ :

$$
G_{4}=a_{6} H_{B} \cdot \sigma\left(\hat{s}_{1}\right)-a_{7}\left[\tilde{S}_{0}^{2}+\left(20 n_{7}-n_{7}^{2}+8 n_{9}-n_{7} n_{9}-92\right) H_{B}^{2}\right] .
$$

Here $a_{6}$ and $a_{7}$ are free discrete parameters entering the $G_{4}$-flux. Their quantization is fixed by the $G_{4}$-flux quantization condition (2.1) using the the expression (3.7) for $c_{2}\left(X_{F_{11}}\right)$. Solving flux quantization in general requires the knowledge of the integral basis for $H_{V}^{(2,2)}\left(X_{F_{11}}\right)$. Since the determination of the general integral basis is beyond the scope of this work, we will check $G_{4}$-flux quantization in dependence on the number of chiral families indirectly by ensuring an integral and positive number $n_{\mathrm{D} 3}$ of D3-branes and quantization of the $3 \mathrm{D}$ CS-terms (2.3). For the detailed discussion, we refer to section 3.3.

In order to compute the $4 \mathrm{D}$ matter chiralities we have to compute the homology classes of the matter surfaces for all representations in table 1. For those codimension two matter surfaces given as complete intersections in the toric ambient space of $X_{F_{11}}$, the homology classes of the corresponding matter surfaces follow directly from the second column in 
table 1 and the splitting of the fiber (3.1) at the respective locus, cf. [22]. We obtain

$$
\begin{aligned}
\mathcal{C}_{(\mathbf{3}, \mathbf{2})_{1 / 6}}^{w} & =\mathcal{S}_{9} \cdot\left(\left[K_{B}^{-1}\right]+\mathcal{S}_{7}-\mathcal{S}_{9}\right) \cdot E_{4}, \\
\mathcal{C}_{(\overline{\mathbf{3}}, \mathbf{1})_{-2 / 3}}^{w} & =-\mathcal{S}_{9} \cdot\left(2\left[K_{B}^{-1}\right]-\mathcal{S}_{7}\right) \cdot\left(2 H-E_{2}-E_{3}-\mathcal{S}_{7}+\mathcal{S}_{9}+\left[K_{B}^{-1}\right]\right), \\
\mathcal{C}_{(\mathbf{1}, \mathbf{1})_{1}}^{w} & =-\left(2\left[K_{B}^{-1}\right]-\mathcal{S}_{7}\right) \cdot\left(3\left[K_{B}^{-1}\right]-\mathcal{S}_{7}-\mathcal{S}_{9}\right) \cdot\left(2 H-E_{1}-E_{4}+\mathcal{S}_{9}\right),
\end{aligned}
$$

where we have used (2.9). Here, we have chosen a node in the respective fiber at codimension two that is not intersected by the zero section. The matter surfaces for the two matter loci supporting the representations $(\mathbf{1}, \mathbf{2})_{-1 / 2}$ and $(\overline{\mathbf{3}}, \mathbf{1})_{1 / 3}$ are not complete intersections. Their associated prime ideals are computed by a primary decomposition. Their respective homology classes are obtained by choosing a suitable complete intersection containing a given matter surface and by subtracting all its other irreducible components with their corresponding multiplicities as determined by the resultant. We obtain

$$
\begin{gathered}
{\left[\mathcal{C}_{(\mathbf{1}, \mathbf{2})_{-1 / 2}}^{w}\right]=\left[\left(D_{1}^{\mathrm{SU}(2)}+D_{1}^{\mathrm{SU}(3)}+2 D_{2}^{\mathrm{SU}(3)}+4\left[K_{B}^{-1}\right]+3 S_{1}-2 \mathcal{S}_{7}\right) \cdot\left(6\left[K_{B}^{-1}\right]-2 \mathcal{S}_{7}-\mathcal{S}_{9}\right)\right.} \\
-2\left(2\left[K_{B}^{-1}\right]-\mathcal{S}_{7}\right) \cdot\left(3\left[K_{B}^{-1}\right]-\mathcal{S}_{7}-\mathcal{S}_{9}\right) \\
\left.+2\left(-2\left[K_{B}^{-1}\right]+\mathcal{S}_{7}\right) \cdot \mathcal{S}_{9}\right] \cdot\left(\mathcal{S}_{9}-\mathcal{S}_{7}-\left[K_{B}^{-1}\right]\right) \\
{\left[\mathcal{C}_{\left(\overline{3}, \mathbf{1}_{1 / 3}\right.}^{w}\right]=\left[2\left[K_{B}^{-1}\right] \cdot\left(\mathcal{S}_{7}-2\left[K_{B}^{-1}\right]\right)+\left(D_{1}^{\mathrm{SU}(2)}+D_{2}^{\mathrm{SU}(3)}+2\left[K_{B}^{-1}\right]+2 S_{1}-\mathcal{S}_{7}\right)\right.} \\
\left.\quad \times\left(5\left[K_{B}^{-1}\right]-\mathcal{S}_{7}-\mathcal{S}_{9}\right)\right] \cdot \mathcal{S}_{9} .
\end{gathered}
$$

Finally, we compute the integrals (2.7) of the $G_{4}$-flux in (3.14) over the various matter surfaces in (3.15) and (3.16), yielding the following 4D matter chiralities:

$$
\begin{aligned}
\chi_{(\mathbf{3}, \mathbf{2})_{1 / 6}} & =\frac{1}{6}\left(4+n_{7}-n_{9}\right) n_{9} a_{6}, \\
\chi_{(\mathbf{1}, \mathbf{2})_{-1 / 2}} & =\frac{1}{2}\left(4+n_{7}-n_{9}\right)\left(\left(2 n_{7}+n_{9}-24\right) a_{6}+4\left(n_{7}-8\right)\left(n_{7}+n_{9}\right) a_{7}-12\right) \\
\chi_{(\overline{\mathbf{3}}, \mathbf{1})_{-2 / 3}} & =\frac{1}{3}\left(n_{7}-8\right) n_{9}\left(2 a_{6}+3\left(n_{7}+n_{9}-12\right) a_{7}\right), \\
\chi_{(\overline{\mathbf{3}}, \mathbf{1})_{1 / 3}} & =-\frac{1}{3} n_{9}\left(\left(n_{7}+n_{9}-20\right) a_{6}+3\left(n_{7}-8\right)\left(n_{7}+n_{9}-12\right) a_{7}\right), \\
\chi_{(\mathbf{1}, \mathbf{1})_{1}} & =\left(n_{7}-8\right)\left(n_{7}+n_{9}-12\right)\left(a_{6}+\left(2 n_{7}+n_{9}-16\right) a_{7}\right) .
\end{aligned}
$$

As a cross-check of these geometric results, we use the duality between M- and F-theory in three dimensions and the implied matching of 3D CS-terms [15, 51, 54] to compute the $4 \mathrm{D}$ chiralities. For the spectrum in table (1), we compute all nonzero CS-terms on the F-theory side as

$$
\begin{aligned}
\Theta_{m=1, n=1}^{\mathrm{F}} & =\frac{1}{2}\left(\frac{1}{6} \chi_{(\mathbf{3}, \mathbf{2})_{1 / 6}}-\frac{1}{2} \chi_{(\mathbf{1}, \mathbf{2})_{-1 / 2}}-\frac{4}{3} \chi_{(\overline{\mathbf{3}}, \mathbf{1})_{-2 / 3}}+\frac{1}{3} \chi_{(\overline{\mathbf{3}}, \mathbf{1})_{1 / 3}}+3 \chi_{(\mathbf{1}, \mathbf{1})_{1}}\right), \\
\Theta_{i=2, j=2}^{\mathrm{F}} & =3 \chi_{(\mathbf{3}, \mathbf{2})_{1 / 6}}-\chi_{(\mathbf{1}, \mathbf{2})_{-1 / 2}}, \\
\Theta_{i=3, j=3}^{\mathrm{F}} & =2 \chi_{(\mathbf{3}, \mathbf{2})_{1 / 6}}-\chi_{(\overline{\mathbf{3}}, \mathbf{1})_{-2 / 3}}+\chi_{(\overline{\mathbf{3}}, \mathbf{1})_{1 / 3}}, \\
\Theta_{i=3, j=4}^{\mathrm{F}} & =-\frac{1}{2}\left(2 \chi_{(\mathbf{3}, \mathbf{2})_{1 / 6}}-\chi_{(\overline{\mathbf{3}}, \mathbf{1})_{-2 / 3}}+\chi_{(\overline{\mathbf{3}}, \mathbf{1})_{1 / 3}}\right) \\
\Theta_{i=4, j=4}^{\mathrm{F}} & =2 \chi_{(\mathbf{3}, \mathbf{2})_{1 / 6}}-\chi_{(\overline{\mathbf{3}}, \mathbf{1})_{-2 / 3}}+\chi_{(\mathbf{3}, \mathbf{1})_{1 / 3}},
\end{aligned}
$$


where the label $m=1$ corresponds to the divisor of the $4 \mathrm{D} \mathrm{U}(1)$ gauge fields, $i=2$ to the Cartan divisor $D_{1}^{\mathrm{SU}(2)}$ and $i=3,4$ to the Cartan divisors $D_{1}^{\mathrm{SU}(3)}$ and $D_{2}^{\mathrm{SU}(3)}$, respectively. We note that only the SM-singlet has a non-trivial KK-charge, cf. [22]. We readily compute the CS-terms (2.3) on the M-theory side for the $G_{4}$-flux (3.14). The matching $\Theta_{A B}^{\mathrm{F}} \stackrel{!}{=} \Theta_{A B}^{\mathrm{M}}$ precisely reproduces the chiralities in (3.17).

\subsection{D anomaly cancelation and family structure}

As an additional cross-check of our computations, we can verify that all 4D anomalies are canceled by a generalized Green-Schwarz mechanism [97, 98].

For $X_{F_{11}}$, we have the following conditions implied by cancelation of the purely non-Abelian, mixed Abelian-non-Abelian, purely Abelian and mixed Abelian-gravitational anomalies:

$$
\begin{aligned}
& \mathrm{SU}(3)^{3}:-2 \chi_{(\mathbf{3}, \mathbf{2})_{1 / 6}}+\chi_{(\overline{\mathbf{3}}, \mathbf{1})_{-2 / 3}}+\chi_{(\overline{\mathbf{3}}, \mathbf{1})_{1 / 3}}=0, \\
& \mathrm{SU}(2)^{2}-\mathrm{U}(1): \frac{1}{2}\left[3 \chi_{(\mathbf{3}, \mathbf{2})_{1 / 6}}\left(\frac{1}{6}\right)+\chi_{(\mathbf{1}, \mathbf{2})_{-1 / 2}}\left(-\frac{1}{2}\right)\right]=-\frac{1}{8} b_{\mathrm{SU}(2)}^{\alpha} \Theta_{\alpha, m=1}, \\
& \mathrm{SU}(3)^{2}-\mathrm{U}(1): \frac{1}{2}\left[2 \chi_{(\mathbf{3}, \mathbf{2})_{1 / 6}}\left(\frac{1}{6}\right)+\chi_{(\overline{\mathbf{3}}, \mathbf{1})_{-2 / 3}}\left(-\frac{2}{3}\right)\right. \\
& \left.+\chi_{(\overline{\mathbf{3}}, \mathbf{1})_{1 / 3}}\left(\frac{1}{3}\right)\right]=-\frac{1}{8} b_{\mathrm{SU}(3)}^{\alpha} \Theta_{\alpha, m=1}, \\
& \mathrm{U}(1)^{3}: \frac{1}{6}\left[6 \chi_{(\mathbf{3 , 2})_{1 / 6}}\left(\frac{1}{6}\right)^{3}+2 \chi_{(\mathbf{1}, \mathbf{2})_{-1 / 2}}\left(-\frac{1}{2}\right)^{3}+3 \chi_{(\overline{\mathbf{3}}, \mathbf{1})_{-2 / 3}}\left(-\frac{2}{3}\right)^{3}\right. \\
& \left.+3 \chi_{(\overline{\mathbf{3}}, \mathbf{1})_{1 / 3}}\left(\frac{1}{3}\right)^{3}+\chi_{(\mathbf{1}, \mathbf{1})_{1}}(1)^{3}\right]=-\frac{1}{8} b_{11}^{\alpha} \Theta_{\alpha, m=1}, \\
& \text { Grav. }^{2}-\mathrm{U}(1): \frac{1}{48}\left[6 \chi_{(\mathbf{3 , 2})_{1 / 6}}\left(\frac{1}{6}\right)+2 \chi_{(\mathbf{1}, \mathbf{2})_{-1 / 2}}\left(-\frac{1}{2}\right)+3 \chi_{(\overline{\mathbf{3}}, \mathbf{1})_{-2 / 3}}\left(-\frac{2}{3}\right)\right. \\
& \left.+3 \chi_{(\overline{\mathbf{3}}, \mathbf{1})_{1 / 3}}\left(\frac{1}{3}\right)+\chi_{(\mathbf{1}, \mathbf{1})_{1}}(1)\right]=\frac{1}{32} a^{\alpha} \Theta_{\alpha, m=1} .
\end{aligned}
$$

We recall that the index $\alpha$ runs over base divisors. For $\mathbb{P}^{3}$ we only have $\alpha=1$ for the single vertical divisor $H_{B}$. The coefficients $b_{11}^{\alpha=1}, b_{\mathrm{SU}(2)}^{\alpha=1}$ and $b_{\mathrm{SU}(3)}^{\alpha=1}$ can be computed as intersections of $H_{B}^{2}$ with the Néron-Tate height pairing $b_{11}$ (3.5), and the GUT divisors $S_{\mathrm{SU}(2)}, S_{\mathrm{SU}(3)}$, respectively. They read, written in terms of the integers $n_{7}, n_{9}$ introduced in (3.8), as

$$
b_{11}^{\alpha=1}=6-\frac{1}{2} n_{7}-\frac{1}{6} n_{9}, \quad b_{\mathrm{SU}(2)}^{\alpha=1}=4+n_{7}-n_{9}, \quad b_{\mathrm{SU}(3)}^{\alpha=1}=n_{9} .
$$

The coefficient $a^{\alpha}$ in (3.19) appearing in the mixed Abelian-gravitational anomaly stems from expanding $K_{B}$ in terms of the vertical divisors. For this particular case we have $a^{\alpha=1}=-4$, according to (3.8). Finally, the CS-term $\Theta_{\alpha=1, m=1}$ for the axion gauging is given by

$$
\Theta_{\alpha=1, m=1}=\frac{1}{6}\left[\left(-36+3 n_{7}+n_{9}\right) a_{6}+6\left(-8+n_{7}\right)\left(-12+n_{7}+n_{9}\right) a_{7}\right],
$$


as we compute using the $G_{4}$-flux in (3.14) and the general formula (2.3). Using these results and the chiralities (3.17), we find that all anomalies in relations (3.19) are indeed satisfied.

Regarding the anomalies there are two remarks in order. First, we recall that even though the pure $\mathrm{SU}(2)$ anomaly is trivial, one has to guarantee that the model does not have a Witten anomaly, i.e. the spectrum of the theory must always exhibit an even number of doublets [99]. In our case, the Witten anomaly takes the form

$$
3 \chi_{(\mathbf{3}, \mathbf{2})_{1 / 6}}+\chi_{(\mathbf{1}, \mathbf{2})_{-1 / 2}} \in 2 \mathbb{Z} .
$$

Using again the expressions given in (3.17), we see that the Witten anomaly is canceled only if $a_{6}\left(n_{7}+n_{9}\right)$ is an even number. This is in contrast to the anomalies (3.19), which are canceled independently of the specific values for $n_{7}, n_{9}, a_{6}$ and $a_{7}$. We expect that (3.22) is automatically obeyed for appropriately quantized $G_{4}$-flux. For a model exhibiting a family structure, which is the case of interest in the following, we have $\chi_{(\mathbf{3}, \mathbf{2})_{1 / 6}}=\chi_{(\mathbf{1 , 2})_{-1 / 2}}$ for which (3.22) is trivially satisfied.

Second, for general $G_{4}$-flux, the axion gauging (3.21) is non-zero, as required by anomalies. This induces a mass-term for the $\mathrm{U}(1)$ gauge field of $X_{F_{11}}$. As the U(1) in this model corresponds to the hypercharge $\mathrm{U}(1)_{\mathrm{Y}}$, we have to impose that it is massless for the sake of the phenomenology of our model. For this reason we have to require $\Theta_{\alpha=1, m=1}=0$, which reduces (3.14) to a one-parameter $G_{4}$-flux. In the absence of the axion gauging all anomalies in (3.19) must vanish identically. Precisely as in the Standard Model, the cancelation of all anomalies can only be achieved if the chiralities for all fields coincide, i.e. if the matter fields come in a certain number $b$ of complete families. Written in terms of this parameter $b \equiv \chi_{(\mathbf{3 , 2})_{1 / 6}}$, the coefficients $a_{6}$ and $a_{7}$ take the following form:

$$
a_{6}=\frac{6 b}{\left(4+n_{7}-n_{9}\right) n_{9}}, \quad a_{7}=-\frac{b\left(3 n_{7}+n_{9}-36\right)}{n_{9}\left(n_{7}-8\right)\left(4+n_{7}-n_{9}\right)\left(n_{7}+n_{9}-12\right)} .
$$

We note that the vanishing of the factors appearing in the denominator of the above equations define the boundaries of the allowed region for $\left(n_{7}, n_{9}\right)$, see figure 2 . Recall also that at some of the boundaries of the allowed region, one of the base divisors supporting a non-Abelian gauge group disappears. For this reasons, the boundary region has to be excluded to begin with since there one or more matter curves disappear, leading to a model unsuited for phenomenological applications.

Using (3.23), we can write the $G_{4}$-flux (3.14) as a function of $b, n_{7}$ and $n_{9}$. In this parametrization, we check, for every allowed value for $\left(n_{7}, n_{9}\right)$, whether there are integral values of $b$ for which the number $n_{\mathrm{D} 3}$ of D3-branes needed to cancel the tadpole (2.2) is a positive integer, as expected for a smooth CY-fourfold $X_{F_{11}}$ and appropriately quantized $G_{4}$-flux [81]. Additionally, we impose that all CS-terms (2.3) are integral, which is equivalent to $G_{4}$-flux quantization as discussed in section 2 . Without adding additional horizontal $G_{4}$-flux, these two conditions impose the lower bounds on the number $b$ of families shown in table 2 for all values of $\left(n_{7}, n_{9}\right)$ in the allowed region and together with the corresponding numbers $n_{\mathrm{D} 3}$ of D3-branes. Remarkably, this simple analysis shows that the minimal value of generations $b$ obeying these constraints is three. We find $b=3$ generations with 


\begin{tabular}{|c|ccccccc|}
\hline$n_{7} \backslash^{n_{9}}$ & 1 & 2 & 3 & 4 & 5 & 6 & 7 \\
\hline 7 & - & $(27 ; 16)$ & - & - & & & \\
6 & - & $(12 ; 81)$ & $(21 ; 42)$ & - & - & & \\
5 & - & - & $(12 ; 57)$ & $(30 ; 8)$ & - & $(3 ; 46)$ & \\
4 & $(42 ; 4)$ & - & $(30 ; 32)$ & - & - & - & - \\
3 & - & $(21 ; 72)$ & - & - & - & $(15 ; 30)$ & \\
2 & $(45 ; 16)$ & $(24 ; 79)$ & $(21 ; 66)$ & $(24 ; 44)$ & $(3 ; 64)$ & & \\
1 & - & - & - & - & & & \\
0 & - & - & $(12 ; 112)$ & & & & \\
-1 & $(36 ; 91)$ & $(33 ; 74)$ & & & & & \\
-2 & - & & & & & & \\
\hline
\end{tabular}

Table 2. The entries $\left(b, n_{\mathrm{D} 3}\right)$ show the minimal number of families $b$ for which the number $n_{\mathrm{D} 3}$ of D3-branes is integral and positive for integral 3D CS-terms. At the allowed points for $\left(n_{7}, n_{9}\right)$ marked as "-" the number of D3-branes is negative for all positive integral values of $b$.

$n_{\mathrm{D} 3}=64$ and $n_{\mathrm{D} 3}=46$ for the two strata with $\left(n_{7}, n_{9}\right)=(2,5)$ and $\left(n_{7}, n_{9}\right)=(5,6)$, respectively. ${ }^{9}$

\subsection{Phenomenological discussion}

The discussion in the previous section shows that only the two models with $\left(n_{7}, n_{9}\right)=$ $(2,5),(5,6)$ admit three chiral families, cf. table 2 . Note also that $b=3$ is the smallest permitted number of generations. Having found these three family solutions, we proceed in this section with the discussion of the phenomenology of the model.

We begin by identifying the representations from table 1 with the Standard Model particles they correspond to:

\begin{tabular}{|c|c|c|c|c|c|}
\hline$Q_{i}$ & $\bar{u}_{i}$ & $\bar{d}_{i}$ & $L_{i}$ & $\bar{e}_{i}$ & $H_{u}, H_{d}$ \\
\hline$(\mathbf{3}, \mathbf{2})_{1 / 6}$ & $(\overline{\mathbf{3}}, \mathbf{1})_{-2 / 3}$ & $(\overline{\mathbf{3}}, \mathbf{1})_{1 / 3}$ & $(\mathbf{1}, \mathbf{2})_{-1 / 2}$ & $(\mathbf{1}, \mathbf{1})_{1}$ & $(\mathbf{1}, \mathbf{2})_{ \pm 1 / 2}$ \\
\hline
\end{tabular}

Here the index $i=1,2,3$ labels the families and we use the common notation to denote quarks by $Q_{i}, \bar{u}_{i}$ and $\bar{d}_{i}$, leptons by $L_{i}$ and $\bar{e}_{i}$ and the two Higgses by $H_{u}$ and $H_{d}$, respectively.

In $X_{F_{11}}$ the Higgs fields emerge as a vector-like pair from the same matter curve as the leptons $L_{i}$. In order to check geometrically that there is indeed a massless vector-like pair supported on the corresponding matter curve we need to be able to go beyond the chiral index and compute the individual numbers of left- and right-chiral fermions for the $G_{4^{-}}$ flux (3.14). Unfortunately, these techniques are not available as of now, see however [100] for promising recent advancements in this direction. Thus, we work in the following under the assumption that the desired vector-like pair is indeed part of the massless spectrum.

\footnotetext{
${ }^{9}$ Adding horizontal $G_{4}$-flux can lower the number of D3-branes further.
} 


\begin{tabular}{|c|c|}
\hline Yukawa & Locus \\
\hline$(\mathbf{3}, \mathbf{2})_{1 / 6} \cdot(\overline{\mathbf{3}}, \mathbf{1})_{-2 / 3} \cdot \overline{(\mathbf{1}, \mathbf{2})_{-1 / 2}}$ & $s_{3}=s_{5}=s_{9}=0$ \\
\hline$(\mathbf{3}, \mathbf{2})_{1 / 6} \cdot(\overline{\mathbf{3}}, \mathbf{1})_{1 / 3} \cdot(\mathbf{1}, \mathbf{2})_{-1 / 2}$ & $s_{3}=s_{9}=0=s_{1} s_{6}-s_{2} s_{5}$ \\
\hline$(\overline{\mathbf{3}}, \mathbf{1})_{-2 / 3} \cdot(\overline{\mathbf{3}}, \mathbf{1})_{1 / 3} \cdot(\mathbf{1}, \mathbf{1})_{1}$ & $s_{1}=s_{5}=s_{9}=0$ \\
\hline$(\mathbf{3}, \mathbf{2})_{1 / 6} \cdot(\mathbf{3}, \mathbf{2})_{1 / 6} \cdot \overline{(\overline{\mathbf{3}}, \mathbf{1})_{1 / 3}}$ & $s_{3}=s_{9}=s_{6}=0$ \\
\hline$(\mathbf{1}, \mathbf{2})_{-1 / 2} \cdot(\mathbf{1}, \mathbf{2})_{-1 / 2} \cdot(\mathbf{1}, \mathbf{1})_{1}$ & $s_{1}=s_{5}=s_{3}=0$ \\
\hline$(\overline{\mathbf{3}}, \mathbf{1})_{1 / 3} \cdot(\overline{\mathbf{3}}, \mathbf{1})_{1 / 3} \cdot(\overline{\mathbf{3}}, \mathbf{1})_{-2 / 3}$ & $s_{5}=s_{6}=s_{9}$ \\
\hline
\end{tabular}

Table 3. Codimension three loci and corresponding Yukawa couplings for $X_{F_{11}}$.

Then it would be possible to induce the following bilinear coupling

$$
\mathcal{W} \subset \mu H_{u} H_{d}+\beta^{i} H_{u} L_{i} .
$$

These two terms could be generated by tuning the complex structure of our model to a model with enhanced (non-Abelian or Abelian) gauge symmetry and a SM-singlet 1, that admits Yukawa couplings with $H_{u}, H_{d}$ and $L_{i}$, respectively. Then if 1 acquires a VEV, which breaks the enhanced gauge symmetry, the superpotential (3.25) could be generated. While the $\mu$-term has to be very small in order to be consistent with electroweak symmetry breaking, the $\beta^{i}$ terms are lepton violating and hence they must be adequately suppressed. We note that both these coefficients are moduli dependent functions, that cannot be computed by known techniques. However, we expect that in a sufficiently generic geometry the moduli of $X_{F_{11}}$ allow for appropriate tunings providing a phenomenologically viable scenario. At this point, we must remark that the geometry of $X_{F_{11}}$ offers no obvious way by which we could assign a quantum number to forbid the $\mu$-term or the $\beta^{i}$ terms.

Regarding the trilinear couplings we note that it was shown in [22] that all gauge invariant trilinear couplings are realized geometrically, see table 3. Thus, all MSSM Yukawas are geometrically allowed, giving rise to the superpotential terms

$$
\mathcal{W} \subset Y_{i, j}^{u} Q_{i} \bar{u}_{j} H_{u}+Y_{i, j}^{d} Q_{i} \bar{d}_{j} H_{d}+Y_{i, j}^{L} \bar{e}_{i} \bar{L}_{j} H_{d} .
$$

Since all three copies of each SM field live on the same matter curve, it is expected that the hierarchies in the Yukawas are generated in a similar fashion as in most SU(5) F-theory GUTs, where the Yukawa matrix has rank one, so that the geometrical coupling gives the mass for the heavy generation while the lighter ones pick their masses from instanton contributions [101-103]

Note also that since we cannot distinguish between $H_{d}$ and $L_{i}$, the following dimension four proton decay operators are also geometrically allowed

$$
\mathcal{W} \subset \lambda_{i, j, k}^{(0)} Q_{i} \bar{d}_{j} L_{k}+\lambda_{i, j, k}^{(1)} \bar{e}_{i} L_{j} L_{k}+\lambda_{i, j, k}^{(2)} \bar{u}_{i} \bar{d}_{j} \bar{d}_{k} .
$$

Here the coupling $\lambda^{(2)}$ can only be suppressed by appropriate tunings of moduli. However, this is not possible for $\lambda^{(0)}, \lambda^{(1)}$ as their geometric origin is the same as that of the 

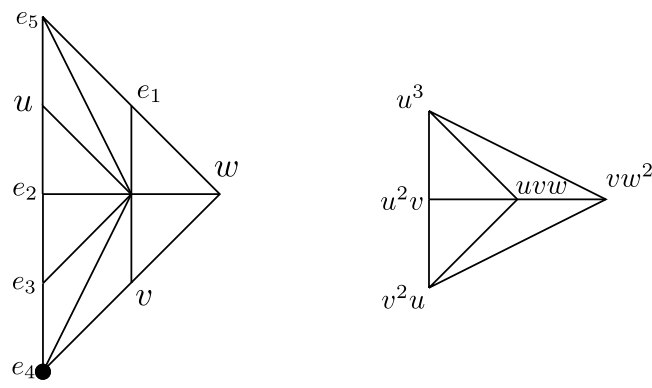

\begin{tabular}{|c|c|}
\hline section & Line Bundle \\
\hline$u$ & $\mathcal{O}\left(H-E_{1}-E_{2}-E_{5}+\mathcal{S}_{9}+\left[K_{B}\right]\right)$ \\
\hline$v$ & $\mathcal{O}\left(H-E_{2}-E_{3}-E_{4}+\mathcal{S}_{9}-\mathcal{S}_{7}\right)$ \\
\hline$w$ & $\mathcal{O}\left(H-E_{1}\right)$ \\
\hline$e_{1}$ & $\mathcal{O}\left(E_{1}-E_{5}\right)$ \\
\hline$e_{2}$ & $\mathcal{O}\left(E_{2}-E_{3}\right)$ \\
\hline$e_{3}$ & $\mathcal{O}\left(E_{3}-E_{4}\right)$ \\
\hline$e_{4}$ & $\mathcal{O}\left(E_{4}\right)$ \\
\hline$e_{5}$ & $\mathcal{O}\left(E_{5}\right)$ \\
\hline
\end{tabular}

Figure 3. The toric diagram of polyhedron $F_{13}$ and its dual. The zero section is indicated by the dot. In the accompanying table we indicate the divisor classes of the fiber coordinates.

Yukawa couplings (3.26). It then seems very challenging to suppress them to orders of $\lambda \leq 10^{-10}$ [104], while keeping the Yukawa couplings at values of about 0.1 .

\section{Pati-Salam model: $G_{F_{13}}=\left(\mathrm{SU}(4) \times \mathrm{SU}(2)^{2}\right) / \mathbb{Z}_{2}$}

The fibration $X_{F_{13}}$ has been shown to exhibit the gauge symmetry and matter representation of the Pati-Salam (PS) model [22]. In section 4.1 we review the geometrical properties of $X_{F_{13}}$. In section 4.2 we explicitly construct $G_{4}$-flux for the base $B=\mathbb{P}^{3}$. There we also compute the homology class of all matter surfaces and the $4 \mathrm{D}$ chiralities for all matter representations. We also determine the minimal number of generations which allow for D3brane tadpole cancelation and integral $G_{4}$-flux. Finally, the phenomenology of F-theoretic PS-models with three generations and their Higgsing down to the MSSM are described in section 4.3.

Readers directly interested in the 4D chiralities and phenomenological aspects of the models can directly jump to (4.14) and the following discussions.

\subsection{The geometry of gauge group and matter representations}

The elliptic fiber which is yields F-theory models that naturally give rise to the gauge group and charge pattern needed for models of Pati-Salam (PS) unification is given by the following CY-hypersurface

$$
p_{F_{13}}=s_{1} e_{1}^{2} e_{2}^{2} e_{3} e_{5}^{4} u^{3}+s_{2} e_{1} e_{2}^{2} e_{3}^{2} e_{4}^{2} e_{5}^{2} u^{2} v+s_{3} e_{2}^{2} e_{3}^{3} e_{4}^{4} u v^{2}+s_{6} e_{1} e_{2} e_{3} e_{4} e_{5} u v w+s_{9} e_{1} v w^{2}
$$

defined in the toric ambient space $\mathbb{P}_{F_{13}}$. The toric diagram of the ambient space as well as the divisor classes of the fiber coordinates are summarized in figure 3 , where as before $H$ is the hyperplane on $\mathbb{P}^{2}$ and $E_{i}, i=1, \ldots, 5$, denote the exceptional divisors.

The elliptically fibered Calabi-Yau fourfold $X_{F_{13}}$ is constructed by promoting the coefficients $s_{i}$ to sections of the line bundles over the base $B$ given in (2.9). The elliptic fibration of $X_{F_{11}}$ is equipped with a zero section given by

$$
\hat{s}_{0}=X_{F_{13}} \cap\left\{e_{4}=0\right\}: \quad\left[1: s_{1}: 1: 1: 1:-s_{9}: 0: 1\right] .
$$




\begin{tabular}{|c|c|}
\hline Representation & Locus \\
\hline$(\mathbf{1}, \mathbf{2}, \mathbf{2})$ & $V\left(I_{(1)}\right):=\left\{s_{1}=s_{3}=0\right\}$ \\
\hline$(\mathbf{4}, \mathbf{2}, \mathbf{1})$ & $V\left(I_{(2)}\right):=\left\{s_{1}=s_{9}=0\right\}$ \\
\hline$(\mathbf{4}, \mathbf{1}, \mathbf{2})$ & $V\left(I_{(3)}\right):=\left\{s_{3}=s_{9}=0\right\}$ \\
\hline$(\mathbf{6}, \mathbf{1}, \mathbf{1})$ & $V\left(I_{(4)}\right):=\left\{s_{6}=s_{9}=0\right\}$ \\
\hline
\end{tabular}

Table 4. Charged matter representations under $\left(\mathrm{SU}(4) \times \mathrm{SU}(2)^{2}\right) / \mathbb{Z}_{2}$ and their associated codimension two loci on $X_{F_{13}}$.

Furthermore, the elliptic fibration admits a section of order two, giving rise to $\mathbb{Z}_{2}$ MordellWeil group [22, 62]. In addition, one can see by computing the discriminant that over the locus $\mathcal{S}_{\mathrm{SU}(4)}=\left\{s_{9}=0\right\}$ the fiber degenerates to an $I_{4}$-fiber. The corresponding Cartan divisors of the resulting $\mathrm{SU}(4)$ gauge symmetry are given by

$$
D_{1}^{\mathrm{SU}(4)}=\left[s_{9}\right]-[u]-\left[e_{2}\right]-\left[e_{3}\right], \quad D_{2}^{\mathrm{SU}(4)}=[u], \quad D_{3}^{\mathrm{SU}(4)}=\left[e_{2}\right] .
$$

Similarly, at the loci $\mathcal{S}_{\mathrm{SU}(2)_{1}}=\left\{s_{1}=0\right\}$ and $\mathcal{S}_{\mathrm{SU}(2)_{2}}=\left\{s_{3}=0\right\}$ we obtain $I_{2}$-fibers. The resulting two $\mathrm{SU}(2)$ factors have the following associated Cartan divisors:

$$
D_{1}^{\mathrm{SU}(2)_{1}}=\left[s_{1}\right]-[v], \quad D_{1}^{\mathrm{SU}(2)_{2}}=\left[e_{1}\right] .
$$

The codimension two loci where the singularities of the fibration enhance, corresponding to the presence of matter fields, are given in table 4 . We readily observe that the codimension two loci of $X_{F_{13}}$ support the matter representations characteristic for the Pati-Salam model. Similar as in section 3.1 we provide base independent expressions for the second Chern class as well as the Euler number of $X_{F_{13}}$, which are needed for $G_{4}$-flux quantization and D3-brane tadpoles. We obtain

$$
\begin{aligned}
c_{2}\left(X_{F_{13}}\right)= & -c_{1}^{2}+c_{2}-6 c_{1} E_{1}-6 E_{1}^{2}-2 c_{1} E_{3}-2 E_{4}^{2}+4 c_{1} H+c_{1} \mathcal{S}_{7}+9 E_{1} \mathcal{S}_{7}+E_{2} \mathcal{S}_{7} \\
& +E_{3} \mathcal{S}_{7}-E_{4} \mathcal{S}_{7}-E_{5} \mathcal{S}_{7}-3 H \mathcal{S}_{7}-4 E_{1} \mathcal{S}_{9}-2 E_{2} \mathcal{S}_{9}+2 H \mathcal{S}_{9} \\
& -2 \mathcal{S}_{7} \mathcal{S}_{9}+2 \mathcal{S}_{9}^{2} \\
\chi\left(X_{F_{13}}\right)= & 12\left(6 c_{1}^{3}+c_{1} c_{2}-4 c_{1}^{2} \mathcal{S}_{7}+2 c_{1} \mathcal{S}_{7}^{2}-6 c_{1}^{2} \mathcal{S}_{9}+2 c_{1} \mathcal{S}_{7} \mathcal{S}_{9}-\mathcal{S}_{7}^{2} \mathcal{S}_{9}+2 c_{1} \mathcal{S}_{9}^{2}\right)
\end{aligned}
$$

where, as before, $c_{1}$ and $c_{2}$ denote the first and second Chern class of the base $B$, respectively, and the divisors $\mathcal{S}_{7}$ and $\mathcal{S}_{9}$ are introduced in (2.9).

Again, we fix the base of the fibration to be $B=\mathbb{P}^{3}$ for the remainder of this section. We expand the divisor $\mathcal{S}_{7}, \mathcal{S}_{9}$ and the anti-canonical class $\left[K_{B}^{-1}\right]$ w.r.t. $H_{B}$ as in 3.8 . Demanding effectiveness of all sections in (2.9) entering the CY-constraint (4.1), we find the allowed values for the pair $\left(n_{7}, n_{9}\right)$ depicted in figure 4 .

\section{2 $G_{4}$-flux and chiral generations}

For the base $B=\mathbb{P}^{3}$, the SR-ideal of the toric ambient space of the fourfold $X_{F_{13}}$ is given by

$$
\begin{gathered}
S R_{F_{13}}=\left\{u e_{1}, u w, u v, u e_{4}, u e_{3}, e_{5} w, e_{5} v, e_{5} e_{4}, e_{5} e_{3}, e_{5} e_{2}, e_{1} v, e_{1} e_{4}, e_{1} e_{3}, e_{1} e_{2},\right. \\
\left.w e_{4}, w e_{3}, w e_{2}, v e_{3}, v e_{2}, e_{4} e_{2}, x_{0} x_{1} x_{2} x_{3}\right\}
\end{gathered}
$$




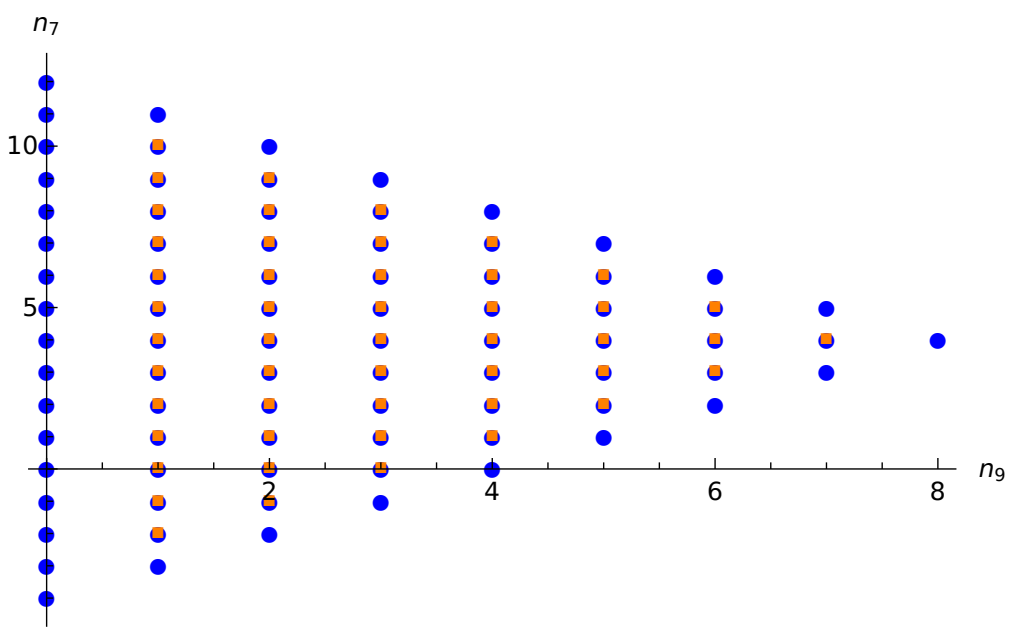

Figure 4. Allowed region of $\left(n_{7}, n_{9}\right)$ for the CY-fourfold $X_{F_{13}}$ with $B=\mathbb{P}^{3}$. For points in orange, all representations of the PS-model are present and a $G_{4}$-flux admitting $b$ families exists.

where, again, $[u, v, w]$ and the $e_{i}, i=1, \ldots, 5$, are the projective coordinates on the fiber and $x_{j}(j=0,1,2,3)$ are the homogeneous coordinates on the $\mathbb{P}^{3}$ base. As a basis of $H^{1,1}\left(X_{F_{13}}\right)$ we choose the hyperplane $H_{B}$, the zero section and the five Cartan divisors,

$$
H^{(1,1)}\left(X_{F_{13}}\right)=\left\langle H_{B}, S_{0}, D_{1}^{\mathrm{SU}(2)_{1}}, D_{1}^{\mathrm{SU}(2)_{2}}, D_{1}^{\mathrm{SU}(4)}, D_{2}^{\mathrm{SU}(4)}, D_{3}^{\mathrm{SU}(4)}\right\rangle,
$$

where, as before, the divisors and their dual $(1,1)$-forms are denoted by the same symbol. Here, $S_{0}$ is the class of $\hat{s}_{0}$.

For the computation of the vertical cohomology ring of $X_{F_{13}}$ we use the SR-ideal (4.7), the basis (4.8) as well as the following intersections,

$$
H_{B}^{3} \cdot S_{0}^{2}=-1, \quad H_{B}^{3} \cdot\left(D_{1}^{\mathrm{SU}(2)_{2}}\right)^{2}=-2, \quad H_{B}^{3} \cdot\left(D_{3}^{\mathrm{SU}(4)}\right)^{2}=-2,
$$

which follow from intersections in $\mathbb{P}_{F_{13}}$. We readily obtain the quartic intersections of $X_{F_{13}}$ and the basis of $H^{(3,3)}\left(X_{F_{13}}\right)$ is canonically determined by (4.8). Considering all products of two divisors in $H^{(1,1)}\left(X_{F_{13}}\right)$, we obtain generators for $H_{V}^{(2,2)}\left(X_{F_{13}}\right)$. The rank of their inner product matrix reveals that

$$
\operatorname{dim} H_{V}^{(2,2)}\left(X_{F_{13}}\right)=8 .
$$

We choose the following eight-dimensional basis for $H_{V}^{(2,2)}\left(X_{F_{13}}\right)$ :

$H_{V}^{(2,2)}\left(X_{F_{13}}\right)=\left\langle H_{B}^{2}, H_{B} S_{0}, H_{B} D_{1}^{\mathrm{SU}(2)_{1}}, H_{B} D_{1}^{\mathrm{SU}(2)_{2}}, H_{B} D_{1}^{\mathrm{SU}(4)}, H_{B} D_{2}^{\mathrm{SU}(4)}, H_{B} D_{3}^{\mathrm{SU}(4)}, S_{0}^{2}\right\rangle$.

Next, we make an ansatz for the $G_{4}$-flux in terms of this basis. Following the description in section 2 we impose the condition (2.6). We find seven conditions on the $G_{4}$-flux which leaves us with the following one parameter flux:

$$
\begin{aligned}
G_{4}=-a_{8}\left[S_{0}^{2}+H_{B} \cdot(\right. & 4 S_{0}+\left(12 n_{9}-n_{7} n_{9}-n_{9}^{2}\right) H_{B}-\frac{1}{2} n_{9} D_{1}^{\mathrm{SU}(2)_{1}} \\
& \left.\left.+\frac{1}{4}\left(n_{7}+n_{9}-12\right)\left(3 D_{1}^{\mathrm{SU}(4)}+2 D_{2}^{\mathrm{SU}(4)}+D_{3}^{\mathrm{SU}(4)}\right)\right)\right] .
\end{aligned}
$$


Here $a_{8}$ is a free discrete parameter, whose quantization is determined by $G_{4}$-flux quantization. As in section 3 we will address $G_{4}$-flux quantization indirectly in dependence on the number of chiral families by ensuring an integral and positive number $n_{\mathrm{D} 3}$ of D3-branes and quantization of 3D CS-terms (2.3). We present the findings of this analysis below.

As a next step, we need the homology classes of all matter surfaces for the representations in table 4. Two of the matter surfaces are given as complete intersections in the toric ambient space $X_{F_{13}}$. Their homology classes are given by

$$
\mathcal{C}_{(\mathbf{4 , 2 , 1})}^{w}=\mathcal{S}_{9} \cdot\left(3\left[K_{B}^{-1}\right]-\mathcal{S}_{7}-\mathcal{S}_{9}\right) \cdot\left(H+\left[K_{B}^{-1}\right]\right), \quad \mathcal{C}_{(\mathbf{4 , 1}, \mathbf{2})}^{w}=\mathcal{S}_{9}\left(\left[K_{B}^{-1}\right]+\mathcal{S}_{7}-\mathcal{S}_{9}\right) E_{5},
$$

where we chose those nodes which are not intersected by the zero section. These matter surfaces lead to the following chiralities

$$
\chi_{(\mathbf{4 , 2 , 1})}=-\chi_{(\mathbf{4 , 1 , 2})}=\frac{1}{4} n_{9}\left(4+n_{7}-n_{9}\right)\left(n_{7}+n_{9}-12\right) a_{8} .
$$

We note that the representations $(\mathbf{1}, \mathbf{2}, \mathbf{2})$ and $(\mathbf{6}, \mathbf{1}, \mathbf{1})$ (see table 4 ) are real representations. Therefore, their chiralities (2.7) are zero by definition. This is manifested in the geometry of $X_{F_{13}}$ by the fact that the fiber at the corresponding codimension two loci is non-split [22], so that precisely the nodes carrying the weights of the respective representation are interchanged by codimension three monodromies. This implies that the integral of the $G_{4}$-flux over the matter surfaces associated to one note is equal to the integral over the matter surface associated to the other node. However, as the sum of the weights of the two nodes has to equal a root of the gauge group, the two integrals add up to zero, see (2.6), and we get zero chirality. It is important to stress that this does not necessarily mean that there are no massless fields transforming under real representations, in F-theory. Indeed, there can be vector-like fields in the theory, which are counted by the number $n(\mathbf{R})$ of individual Weyl fermions. For the above reasons, however, the multiplicity of fermions in real representations is not accessible by the methods described in this work. It would be interesting to develop methods based on those introduced in [100] to compute the number $n(\mathbf{R})$ for real representations explicitly.

We note that according to (4.14), the chiralities of the two complex representations $(4,2,1)$ and $(4,1,2)$ are equal up to a sign, which guarantees the cancelation of the cubic $\mathrm{SU}(4)$ anomaly. ${ }^{10}$ Thus, the number $b$ of chiral generations coincides with the chirality of one of these two representations, i.e. we set $b=\chi_{(\mathbf{4 , 2 , 1})}$. This allows us to express the parameter $a_{8}$ in the $G_{4}$-flux in (4.12) as

$$
a_{8}=\frac{4 b}{n_{9}\left(n_{9}-n_{7}-4\right)\left(12-n_{7}-n_{9}\right)} .
$$

We note again that those values for $\left(n_{7}, n_{9}\right)$, for which the denominator vanishes, are excluded by the requirement to keep all non-Abelian gauge factors and to have non-vanishing

\footnotetext{
${ }^{10}$ Note that the cubic SU(4) anomaly, together with the Witten anomaly of the $\mathrm{SU}(2)$ factors are the only anomalies to check in this model. One can also see that the cancelation of the SU(4) anomaly guarantees that the Witten anomaly cancels as well.
} 


\begin{tabular}{|c|ccccccc|}
\hline$n_{7} \backslash^{n_{9}}$ & 1 & 2 & 3 & 4 & 5 & 6 & 7 \\
\hline 10 & $(13 ; 204)$ & & & & & & \\
9 & - & $(11 ; 140)$ & & & & & \\
8 & $(33 ; 94)$ & $(10 ; 119)$ & $(9 ; 90)$ & & & & \\
7 & - & $(9 ; 100)$ & $(6 ; 77)$ & $(14 ; 48)$ & & & \\
6 & $(15 ; 108)$ & $(8 ; 86)$ & $(21 ; 52)$ & $(12 ; 46)$ & $(5 ; 44)$ & & \\
5 & $(6 ; 106)$ & $(35 ; 44)$ & - & $(30 ; 16)$ & - & $(3 ; 44)$ & \\
4 & $(7 ; 102)$ & $(6 ; 75)$ & $(15 ; 50)$ & $(8 ; 42)$ & $(15 ; 30)$ & $(6 ; 41)$ & $(7 ; 42)$ \\
3 & $(6 ; 106)$ & $(35 ; 44)$ & - & $(30 ; 16)$ & - & $(3 ; 44)$ & \\
2 & $(15 ; 108)$ & $(8 ; 86)$ & $(21 ; 52)$ & $(12 ; 46)$ & $(5 ; 44)$ & & \\
1 & - & $(9 ; 100)$ & $(6 ; 77)$ & $(14 ; 48)$ & & & \\
0 & $(33 ; 94)$ & $(10 ; 119)$ & $(9 ; 90)$ & & & & \\
-1 & - & $(11 ; 140)$ & & & & & \\
-2 & $(13 ; 204)$ & & & & & & \\
\hline
\end{tabular}

Table 5. The entries $\left(b ; n_{\mathrm{D} 3}\right)$ show the minimal number of families $b$ for which the number of D3 branes $n_{D 3}$ is integral and positive for integral 3D CS terms. At the points marked with "-" the number of D3 branes is negative for all positive integral values of $b$.

chiralities (4.14). Thus, we are restricted to the interior (orange dots) of the allowed region for the fibration over $\mathbb{P}^{3}$ in figure 4.

As in section 3.3, we parametrize the $G_{4}$-flux in terms of the integral number $b$ in (4.15) of families. In this parametrization we evaluate the D3-brane tadpole (2.2) and require integral, positive $n_{\mathrm{D} 3}$ and integral CS-terms (2.3). Without further horizontal $G_{4}$-flux, this yields the lower bounds on the number $b$ in dependence of $\left(n_{7}, n_{9}\right)$ shown in table 5 , where also the corresponding numbers $n_{\mathrm{D} 3}$ of D3-branes are displayed. We find that three families are possible at two values for $n_{7}$ and $n_{9}$. Again, we find that in the context of this simple analysis three is the minimal number $b$ of families compatible with the D3-brane tadpole (2.2). We also note that table 5 has inherited the symmetries of the polyhedron $F_{14}$.

We conclude by noting that we double-check the chiralities in (4.14) using the matching (2.5) of CS-terms. The real representations in table 4 do not contribute to the loopcorrections in (2.4). We obtain the non-vanishing CS-terms

$$
\Theta_{i, i}^{\mathrm{F}}=-2 \chi_{\mathbf{4}, \mathbf{1}, \mathbf{2}}(i=1, \ldots, 5), \quad \Theta_{i=3, j=4}^{\mathrm{F}}=\Theta_{i=4, j=5}^{\mathrm{F}}=-\chi_{\mathbf{4}, \mathbf{1}, \mathbf{2}},
$$

where the indices label the five Cartan divisor $\left\{D_{1}^{\mathrm{SU}(2)_{1}}, D_{1}^{\mathrm{SU}(2)_{2}}, D_{1}^{\mathrm{SU}(4)}, D_{2}^{\mathrm{SU}(4)}, D_{3}^{\mathrm{SU}(4)}\right\}_{i}$. Equating this with the CS-terms (2.3) on the M-theory side we readily reproduces (4.14).

\subsection{Phenomenological discussion}

In table 5 we find only two models with $\left(n_{7}, n_{9}\right)=(5,6),(3,6)$ that allow for three chiral Pati-Salam families. As in the standard model we see that three is the minimum allowed number of generations. These two models are equivalent under the reflection along the 
$\left(4, n_{9}\right)$ line, which reflects the invariance of the theory under exchange of the two $\mathrm{SU}(2)$ gauge groups.

The Higgs transition from $X_{F_{13}}$ to $X_{F_{11}}$ has been considered for the six dimensional case [22]. However, some of the observations made there immediately carry over to four dimensions. Similar to the $6 \mathrm{D}$ case, the transition happens due to a toric blow-down in the ambient space of the elliptic fiber of $X_{F_{13}}$. In this case we see that blowing down either $e_{4}$ or $e_{5}$ in figure 3 leads to the toric diagram of $F_{11}$ in figure 1. These two transitions are equivalent up to redefinitions of the coordinates on the fiber, and for this reason we focus only on the blow down of $e_{4}$ which leads to $F_{11}$ in its canonical form.

There are some subtleties that have to be discussed before proceeding with the detailed discussion of the Higgsings. First, we note that the blow-down process requires a restriction of the allowed region in figure 4 for $X_{F_{13}}$ because the section $s_{5}$ has to be effective, which is present in the CY-equation (3.1) for $X_{F_{11}}$, but not in the one (4.1) for $X_{F_{13}}$. This reduction amounts to excluding only two models below the $\left(8, n_{9}\right)$ line in table 9 . To understand from an effective field theory point view, why the Higgsing is not possible for these two models is elusive. We recall that in the six dimensional case, the exclusion of certain points in the allowed region for $\left(n_{7}, n_{9}\right)$ is explained from the field theory point of view by the fact that these models lack a sufficient number of Higgses for carrying out a D-flat Higgsing. In four dimensions one expects the same to occur. However, here the Higgses are pairs of vector-like fields and due to our lack of control over this sector of the theory, we can not verify this statement at this point.

Second, we also note that while in $X_{F_{11}}$ certain points in table 2 do not permit a family structure, in $X_{F_{13}}$ some of these points do allow for a certain number of families. Since the net chirality is preserved by Higgsings, it remains the question of why it is not possible to have the Higgsed models from $X_{F_{13}}$ as consistent tadpole canceling solutions ${ }^{11}$ in $X_{F_{11}}$. It is expected that this seeming contradiction can be resolved by the inclusion of horizontal $G_{4}$-flux. For example, it has been argued in $[62,85]$, at least in simple situations, that horizontal $G_{4}$-flux exists that compensates for the change of the Euler number of a CYfourfold in an extremal transition. Adding this $G_{4}$-flux to F-theory on $X_{F_{11}}$, its D3-brane tadpole (2.2) becomes effectively identical to that on $X_{F_{13}}$ and we expect that our search strategy for three family models on both $X_{F_{11}}$ and $X_{F_{13}}$ will be consistent with the Higgs effect relating their effective actions.

In the following, we consider the special point $\left(n_{7}, n_{9}\right)=(5,6)$ which has three as the smallest number of families both in $X_{F_{11}}$ and $X_{F_{13}}$. Therefore, the toric Higgsing is possible without the necessity of adding further $G_{4}$-flux. ${ }^{12}$ We discuss the field theoretical Higgsing in more detail and make some remarks about the phenomenology of this threefamily model.

\footnotetext{
${ }^{11}$ A logically possible explanation is again the absence of enough Higgs fields for performing the transition to begin with, which can not be tested with the tools at hand. The analysis of the vector-like spectrum using e.g. techniques available at weak coupling is beyond the scope of this work.

${ }^{12}$ Note that the point $\left(n_{7}, n_{9}\right)=(2,5)$ allows for three families in $X_{F_{11}}$, while in $X_{F_{13}}$ the smallest number is five. Hence, based on these simple arguments the Higgsing is not possible without discussing horizontal $G_{4}$-flux.
} 


\begin{tabular}{|c|ccccc|}
\hline Name & Representation & \multicolumn{4}{|c|}{ SM decomposition } \\
\hline PS Higgs & $1 \times(\overline{\mathbf{4}}, \mathbf{1}, \mathbf{2})_{H^{1}}$ & $\rightarrow$ & $\bar{d}_{H}:(\overline{\mathbf{3}}, \mathbf{1})_{\frac{1}{3}}$, & $\bar{u}_{H}:(\overline{\mathbf{3}}, \mathbf{1})_{-\frac{2}{3}}, \bar{e}_{H}:(\mathbf{1}, \mathbf{1})_{1}, \bar{\nu}_{H}:(\mathbf{1}, \mathbf{1})_{0}$ \\
PS Higgs & $1 \times(\mathbf{4}, \mathbf{1}, \mathbf{2})_{H^{2}}$ & $\rightarrow$ & $d_{H}:(\mathbf{3}, \mathbf{1})_{-\frac{1}{3}}, u_{H}:(\mathbf{3}, \mathbf{1})_{\frac{2}{3}}, \quad e_{H}:(\mathbf{1}, \mathbf{1})_{-1}, \nu_{H}:(\mathbf{1}, \mathbf{1})_{0}$ \\
Exotic & $1 \times(\mathbf{6}, \mathbf{1}, \mathbf{1})$ & $\rightarrow$ & $\bar{D}:(\overline{\mathbf{3}}, \mathbf{1})_{\frac{1}{3}}$, & $D:(\mathbf{3}, \mathbf{1})_{-\frac{1}{3}}$ & \\
\hline SM Higgs & $1 \times(\mathbf{1}, \mathbf{2}, \mathbf{2})_{H}$ & $\rightarrow$ & $H_{u}:(\mathbf{1}, \mathbf{2})_{1 / 2}, H_{d}:(\mathbf{1}, \mathbf{2})_{-\frac{1}{2}}$ & \\
SM Matter & $3 \times(\mathbf{4}, \mathbf{2}, \mathbf{1})_{M}$ & $\rightarrow$ & $Q_{i}:(\mathbf{3}, \mathbf{2})_{\frac{1}{6}}$, & $L_{i}:(\mathbf{1}, \mathbf{2})_{-\frac{1}{2}}$ & \\
SM Matter & $3 \times(\overline{\mathbf{4}}, \mathbf{1}, \mathbf{2})_{M}$ & $\rightarrow$ & $\bar{d}:(\overline{\mathbf{3}}, \mathbf{1})_{\frac{1}{3}}$, & $\bar{u}:(\overline{\mathbf{3}}, \mathbf{1})_{-\frac{2}{3}}, \quad \bar{e}:(\mathbf{1}, \mathbf{1})_{1}, \quad \bar{\nu}:(\mathbf{1}, \mathbf{1})_{0}$ \\
\hline
\end{tabular}

Table 6. The Pati-Salam matter content and its decomposition into standard model fields. The MSSM spectrum originates purely from the PS representations of the last three rows, which also provide candidates for right handed neutrinos $\nu$. The representation in the first three rows are the Pati-Salam Higgses and the sextet needed to decouple exotic triplets.

On the field theory side the Higgsing is triggered by a VEV in the $(\mathbf{4}, \mathbf{1}, \mathbf{2})$ representation at the locus $s_{3}=s_{9}=0$. A supersymmetric Higgsing requires at least one vector-like pair of fields in the representation $(\mathbf{4}, \mathbf{1}, \mathbf{2})$ in addition to the three chiral families. However, as mentioned already, we can not determine the massless vector-like spectrum with the techniques presented in section 2. Therefore, we work under the assumption that this vector-like pair of Higgs fields is indeed part of the spectrum. In table 6 we summarize the desired spectrum of the PS-model and its decomposition in terms of representations under the SM gauge group. In addition to the three chiral pairs $(\overline{\mathbf{4}}, \mathbf{1}, \mathbf{2})_{M},(\mathbf{4}, \mathbf{2}, \mathbf{1})_{M}$, we require the presence of a light vector-like pair $(\overline{\mathbf{4}}, \mathbf{1}, \mathbf{2})_{H^{1}}$ and $(\mathbf{4}, \mathbf{1}, \mathbf{2})_{H^{2}}$ to serve as the PS-Higgses, whose neutral components develop VEVs $\left\langle H^{1}\right\rangle=\left\langle H^{2}\right\rangle$. In this type of breaking the hypercharge generator of the SM corresponds to the combination

$$
Q_{Y}=\frac{2}{\sqrt{6}} T_{4}^{15}-T_{2}^{3}
$$

of the broken Cartans $T_{4}^{15}=\frac{1}{2 \sqrt{6}} \operatorname{diag}(1,1,1,-3)$ in $\mathrm{SU}(4)$ and $T_{2}^{3}=\frac{\sigma^{3}}{2}$ in $\mathrm{SU}(2)$. Note also that in addition to these fields, we also expect a sextet and a bidoublet to be part of the massless spectrum. ${ }^{13}$ From the bidoublet $(\mathbf{1}, \mathbf{2}, \mathbf{2})_{H}$ we get the SM-Higgses and from the $(\mathbf{6}, \mathbf{1}, \mathbf{1})$ we get a pair of color triplets which serve to decouple some otherwise massless fields arising from the PS-Higgs multiplet [105].

Regarding the decomposition of the Higgs fields $H^{1}$ and $H^{2}$ in table 6 , we note that the fields $\bar{u}_{H}, u_{H}, \bar{e}_{H}, e_{H}$ are removed from the massless spectrum as half of them become the longitudinal modes of the massive bosons of the broken $\mathrm{SU}(4) \times \mathrm{SU}(2)$ and the other half become massive Higgs bosons [106]. Therefore one only has to care about the lifting of the states $\bar{d}_{H}$ and $d_{H}$. For that purpose we use the exotic $(\mathbf{6}, \mathbf{1}, \mathbf{1})$. In table 7 we present the geometrically allowed Yukawa couplings in $X_{F_{13}}$. Writing the couplings involving the

\footnotetext{
${ }^{13}$ We recall again that the presence of real representations and of a single vector-like pair of PS-Higgses is just introduced as an optimistic possibility in our discussion. It is to be seen if the hypersurface fibration over $\mathbb{P}^{3}$ actually allows for these desired fields.
} 


\begin{tabular}{|c|c|}
\hline Yukawa & Locus \\
\hline$(\mathbf{1 , 2 , 2}) \cdot \overline{(\mathbf{4}, \mathbf{2}, \mathbf{1})} \cdot(\mathbf{4}, \mathbf{1}, \mathbf{2})$ & $s_{1}=s_{3}=s_{9}=0$ \\
\hline$\overline{(\mathbf{6}, \mathbf{1}, \mathbf{1})} \cdot(\mathbf{4}, \mathbf{2}, \mathbf{1}) \cdot(\mathbf{4}, \mathbf{2}, \mathbf{1})$ & $s_{1}=s_{6}=s_{9}=0$ \\
\hline$\overline{(\mathbf{6 , 1}, \mathbf{1})} \cdot(\mathbf{4}, \mathbf{1}, \mathbf{2}) \cdot(\mathbf{4}, \mathbf{1}, \mathbf{2})$ & $s_{3}=s_{6}=s_{9}=0$ \\
\hline
\end{tabular}

Table 7. Codimension three loci and corresponding Yukawa couplings for $X_{F_{13}}$.

sextet and the Higgses and decomposing them into SM representations we find

$$
\left\langle H^{1}\right\rangle \bar{d}_{H} D+\left\langle H^{2}\right\rangle \bar{D} d_{H} \subset(\mathbf{6}, \mathbf{1}, \mathbf{1}) \cdot(\overline{\mathbf{4}}, \mathbf{1}, \mathbf{2})_{H^{1}}^{2}+(\mathbf{6}, \mathbf{1}, \mathbf{1}) \cdot(\mathbf{4}, \mathbf{1}, \mathbf{2})_{H^{2}}^{2} .
$$

With these couplings, the masses of all exotics can be pushed towards the grand unification scale $\Lambda_{\mathrm{PS}}$.

For the real representations we generically expect the following field theoretical bilinears in the superpotential

$$
\mathcal{W} \supset M_{2}(\mathbf{1}, \mathbf{2}, \mathbf{2})_{H} \cdot(\mathbf{1}, \mathbf{2}, \mathbf{2})_{H}+M_{6}(\mathbf{6}, \mathbf{1}, \mathbf{1}) \cdot(\mathbf{6}, \mathbf{1}, \mathbf{1}) .
$$

Similar to the bilinear couplings (3.25) in the SM, these terms are expected to be generated by a VEV of a PS-singlet, that can be made visible by tuning the complex structure of $X_{F_{13}} \cdot{ }^{14}$ Thus, the masses $M_{2}$ and $M_{6}$ are expected to be generically above the PS unification scale. However, after decomposing the bidoublets in terms of the SM gauge group, we see that the mass $M_{2}$ is related to the mass coefficient in front of the bilinear $H_{u} H_{d}$ in (3.25). Therefore, some fine tuning is needed in order to guarantee that the $\mu$ term is small enough. Similarly, the coupling $M_{6}$ enforces a kind of see-saw mechanism for the triplets $d_{H}$ and $D$. The lowest mass eigenstate would have a mass of the order $\Lambda_{\mathrm{PS}}^{2} / M_{6}$. Hence, we have to guarantee that $M_{6}$ is not too far beyond the PS scale as otherwise the exotic masses will be pushed towards observable mass scales. Therefore, we have to rely on the possibility that certain points in the complex structure moduli space of $X_{F_{13}}$ allow for a configuration in which both $M_{2}$ and $M_{6}$ are sufficiently small.

The Yukawa couplings for the SM fields are also generated geometrically as one can see from table 7 . Indeed, we have the couplings

$$
\mathcal{W} \supset(\mathbf{1}, \mathbf{2}, \mathbf{2})_{H} \cdot(\mathbf{4}, \mathbf{2}, \mathbf{1})_{M, i} \cdot(\overline{\mathbf{4}}, \mathbf{1}, \mathbf{2})_{M, j} .
$$

Since both up-and down-type Yukawas arise from the same Yukawa point, the masses for up and down type quarks coincide at the PS-scale. The observed mass splitting is then due to the RG-running of the masses to the infrared. Again, since all matter fields of the same type arise from the same matter curve, the rank of the Yukawa matrix is expected to be one, with the lighter families picking their masses from instanton effects.

We conclude with a final important remark about the Pati-Salam model. We note that Higgses and leptons arise from different representations of the PS-group, which is

\footnotetext{
${ }^{14}$ In fact, we can confirm, that there are codimension three components in the matter curves of the real representations of the PS-model, that could geometrically support the couplings (4.19).
} 

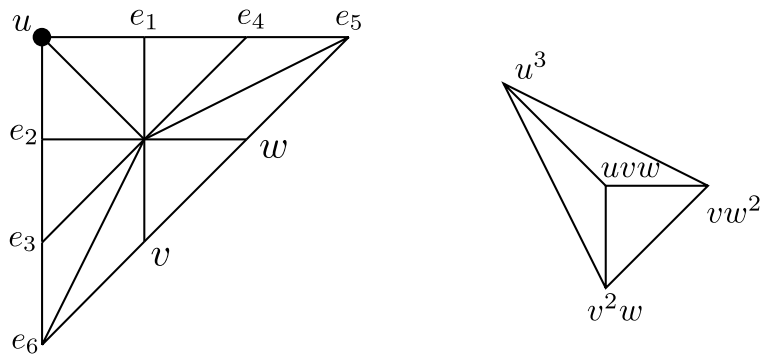

\begin{tabular}{|c|c|}
\hline section & Divisor class \\
\hline$u$ & $\mathcal{O}\left(H-E_{1}-E_{2}+\mathcal{S}_{9}+\left[K_{B}\right]\right)$ \\
\hline$v$ & $\mathcal{O}\left(H-E_{2}-E_{3}-E_{6}+\mathcal{S}_{9}-\mathcal{S}_{7}\right)$ \\
\hline$w$ & $\mathcal{O}\left(H-E_{1}-E_{4}-E_{5}\right)$ \\
\hline$e_{1}$ & $\mathcal{O}\left(E_{1}-E_{4}\right)$ \\
\hline$e_{2}$ & $\mathcal{O}\left(E_{2}-E_{3}\right)$ \\
\hline$e_{3}$ & $\mathcal{O}\left(E_{3}-E_{6}\right)$ \\
\hline$e_{4}$ & $\mathcal{O}\left(E_{4}-E_{5}\right)$ \\
\hline$e_{5}$ & $\mathcal{O}\left(E_{5}\right)$ \\
\hline$e_{6}$ & $\mathcal{O}\left(E_{6}\right)$ \\
\hline
\end{tabular}

Figure 5. The toric diagram of polyhedron $F_{16}$ and its dual. The zero section is indicated by the dot. In the accompanying table we indicate the divisor classes of the fiber coordinates.

in contrast to GUT schemes such based on $\mathrm{SO}(10)$ or $\mathrm{SU}(5)$. This has the advantage that in PS-models, there is no Yukawa coupling which induces the dimension four proton decay operators (3.27), which are only generated below $\Lambda_{\mathrm{PS}}$ after integrating out the heavy triplets. Therefore, these couplings are suppressed by a factor $\Lambda_{\mathrm{PS}} / M_{6}$. The dimension five proton decay operators are generated in a similar fashion. To see this in more detail let us consider the couplings of the SM fields to the exotic sextet

$$
\begin{aligned}
& (\mathbf{6}, \mathbf{1}, \mathbf{1})(\overline{\mathbf{4}}, \mathbf{1}, \mathbf{2})_{M, i}(\overline{\mathbf{4}}, \mathbf{1}, \mathbf{2})_{M, j} \supset \bar{D} \bar{d}_{i} \bar{u}_{j}+D \bar{d}_{i} \nu_{j}+D \bar{u}_{i} \bar{e}_{j}, \\
& (\mathbf{6}, \mathbf{1}, \mathbf{1})(\mathbf{4}, \mathbf{2}, \mathbf{1})_{M, i}(\mathbf{4}, \mathbf{2}, \mathbf{1})_{M, j} \supset \bar{D} Q_{i} L_{j}+D Q_{i} Q_{j} .
\end{aligned}
$$

Upon integration of the exotic states $D, \bar{D}$ we obtain the effective five operators,

$$
Q_{i} Q_{j} Q_{k} L_{m}+Q_{i} L_{j} \bar{u}_{k} \bar{e}_{i}+\bar{d}_{i} \bar{u}_{j} \bar{u}_{k} \bar{e}_{m}
$$

all of which are suppressed by a factor $\left(1 / M_{6}^{2}\right)$.

\section{$5 \quad$ Trinification model: $G_{F_{16}}=\left(\mathrm{SU}(3)^{3}\right) / \mathbb{Z}_{3}$}

The last type of models we consider are F-theory compactifications on $X_{F_{16}}$, which exhibit the gauge group of Trinification as well as its characteristic bitriplet spectrum. The geometrical information relevant for the discussion of the matter and gauge content of the theory is provided in section 5.1. The construction of the $G_{4}$-flux for this models and the induced 4D matter chiralities is discussed in section 5.2. There we also determine the smallest allowed numbers of families for all of the allowed strata in moduli space. Finally, in section 5.3 we focus on a particular model which allows for three generations. We describe the Higgsings to the F-theoretic SM obtained from $X_{F_{11}}$, both on the geometry and field theory sides, and comment on the phenomenology of the model.

Readers directly interested in the 4D matter chiralities and the subsequent phenomenological discussion can start reading at (5.13).

\subsection{The geometry of gauge group and matter representations}

The elliptic fiber used for an F-theoretic realization of the Trinification model is a toric CY-hypersurface in the toric ambient space $\mathbb{P}_{F_{16}}$ given by

$$
p_{F_{16}}=s_{1} e_{1}^{2} e_{2}^{2} e_{3} e_{4} u^{3}+s_{6} e_{1} e_{2} e_{3} e_{4} e_{5} e_{6} u v w+s_{7} e_{2} e_{3}^{2} e_{6}^{3} v^{2} w+s_{9} e_{1} e_{4}^{2} e_{5}^{3} v w^{2} .
$$




\begin{tabular}{|c|c|}
\hline Representation & Locus \\
\hline$(\mathbf{3}, \overline{\mathbf{3}}, \mathbf{1})$ & $V\left(I_{(1)}\right):=\left\{s_{1}=s_{7}=0\right\}$ \\
\hline$(\mathbf{3}, \mathbf{1}, \overline{\mathbf{3}})$ & $V\left(I_{(2)}\right):=\left\{s_{1}=s_{9}=0\right\}$ \\
\hline$(\mathbf{1}, \mathbf{3}, \overline{\mathbf{3}})$ & $V\left(I_{(3)}\right):=\left\{s_{7}=s_{9}=0\right\}$ \\
\hline
\end{tabular}

Table 8. Charged matter representations under $\mathrm{SU}(3)^{3} / \mathbb{Z}_{3}$ and corresponding codimension two loci in $X_{F_{16}}$.

The relevant toric data is provided in figure 5. The divisor classes on the fiber are the hyperplane $H$ and the exceptional divisors $E_{1}$ to $E_{5}$.

In constructing elliptic fibrations $X_{F_{16}}$ with the curve (5.1) we promote the coefficients $s_{i}$ to sections of the line bundles over the base $B$ given in (2.9). The elliptic fibration of $X_{F_{16}}$ exhibits three toric sections with two torsional relations among them, so that the Mordell-Weil group of the fibration is $\mathbb{Z}_{3}[22,62]$. The fibration admits a zero section that we choose as

$$
\hat{s}_{0}=X_{F_{16}} \cap\{u=0\}: \quad\left[0: 1: 1: s_{7}:-s_{9}: 1: 1: 1: 1\right] .
$$

As can be seen by inspecting the Weierstrass model of $X_{F_{16}}$, there are three codimension one loci over which the fiber becomes singular, namely $\mathcal{S}_{\mathrm{SU}(3)^{1}}=\left\{s_{1}=0\right\}, \mathcal{S}_{\mathrm{SU}(3)^{2}}=\left\{s_{7}=0\right\}$ and $\mathcal{S}_{\mathrm{SU}(3)^{3}}=\left\{s_{9}=0\right\}$. The fiber degenerates to $I_{3}$ over these three divisors, giving rise to gauge group $\mathrm{SU}(3)^{3} / \mathbb{Z}_{3}$ which is characteristic of the Trinification model. The MW-torsion $\mathbb{Z}_{3}$ acts simultaneous on the centers of the SU(3) factors. The Cartan divisors for the three $\mathrm{SU}(3)$ factors read

$$
\begin{array}{ll}
D_{1}^{\mathrm{SU}(3)_{1}}=[v], & D_{2}^{\mathrm{SU}(3)_{1}}=[w], \\
D_{1}^{\mathrm{SU}(3)_{2}}=\left[e_{4}\right], & D_{2}^{\mathrm{SU}(3)_{2}}=\left[s_{7}\right]-\left[e_{1}\right]-\left[e_{4}\right], \\
D_{1}^{\mathrm{SU}(3)_{3}}=\left[e_{3}\right], & D_{2}^{\mathrm{SU}(3)_{3}}=\left[s_{9}\right]-\left[e_{2}\right]-\left[e_{3}\right] .
\end{array}
$$

At codimension two we find three loci in $B$, over which the singularity type of the elliptic fibration enhances and bifundamental matter is supported, ${ }^{15}$ see table 8 .

We complete the base independent analysis of $X_{F_{16}}$ with the computation of its second Chern Class as well as its Euler number. Using the methods of [61], we obtain

$$
\begin{aligned}
c_{2}\left(X_{F_{16}}\right)= & -c_{1}^{2}+c_{2}-7 c_{1} E_{1}+2 c_{1} E_{2}-9 E_{4}^{2}-2 c_{1} E_{5}-2 c_{1} E_{6}+4 c_{1} H+3 c_{1} \mathcal{S}_{7} \\
& +5 E_{1} \mathcal{S}_{7}+E_{2} \mathcal{S}_{7}+E_{3} \mathcal{S}_{7}+7 E_{4} \mathcal{S}_{7}+E_{6} \mathcal{S}_{7}-5 H \mathcal{S}_{7}-2 c_{1} \mathcal{S}_{9}+E_{1} \mathcal{S}_{9} \\
& -4 E_{2} \mathcal{S}_{9}-2 E_{3} \mathcal{S}_{9}-8 E_{4} \mathcal{S}_{9}+E_{5} \mathcal{S}_{9}+4 H \mathcal{S}_{9}-4 \mathcal{S}_{7} \mathcal{S}_{9}+4 \mathcal{S}_{9}^{2} \\
\chi\left(X_{F_{16}}\right)= & 3\left(24 c_{1}^{3}+4 c_{1} c_{2}-24 c_{1}^{2} \mathcal{S}_{7}+8 c_{1} \mathcal{S}_{7}^{2}-24 c_{1}^{2} \mathcal{S}_{9}+17 c_{1} \mathcal{S}_{7} \mathcal{S}_{9}\right. \\
& \left.-3 \mathcal{S}_{7}^{2} \mathcal{S}_{9}+8 c_{1} \mathcal{S}_{9}^{2}-3 \mathcal{S}_{7} \mathcal{S}_{9}^{2}\right)
\end{aligned}
$$

\footnotetext{
${ }^{15}$ The effect of the $\mathbb{Z}_{3}$ torsion is manifest at codimension two as the only representations which are manifest are singlets under torsion.
} 


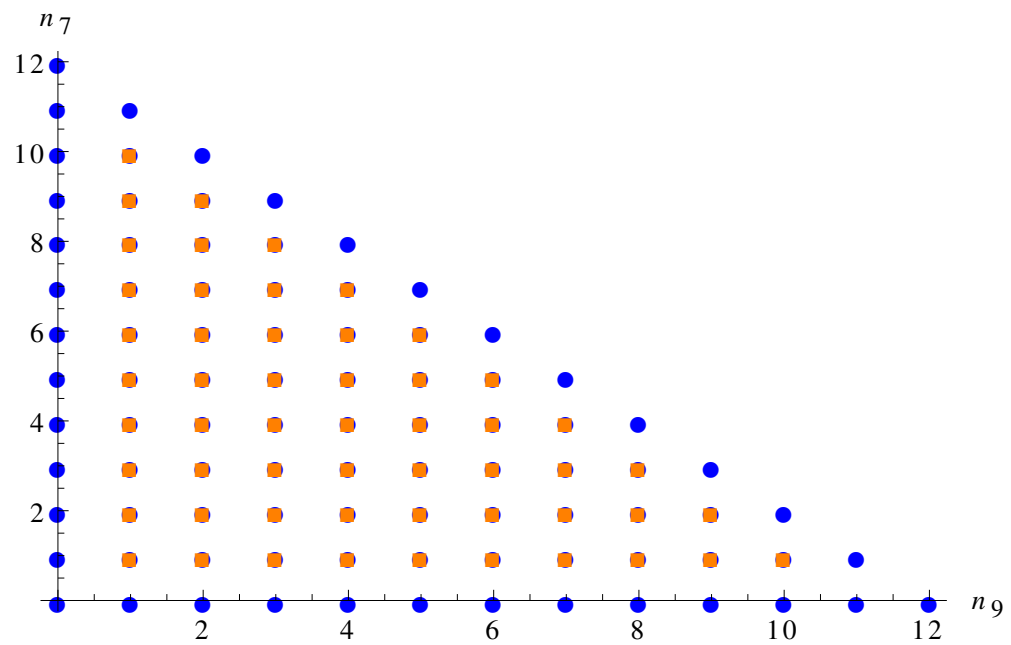

Figure 6. The allowed region $\left(n_{7}, n_{9}\right)$ for the CY-fourfold $X_{F_{16}}$ for all points that are not on the boundary (orange) allow for $b$ families in the Trinification model.

where, as before, $c_{1}$ and $c_{2}$ denoted the first and second Chern class of $B$, respectively.

Next we choose the base $B=\mathbb{P}^{3}$ and expand the divisors $\mathcal{S}_{7}$ and $\mathcal{S}_{9}$ in terms of the pullback of the hyperplane class $H_{B}$, cf. (3.8). Then we use conditions implied by effectiveness of the section $s_{i}$ in (2.9), that enter the CY-equation (5.1), to obtain the allowed region for the pair $\left(n_{7}, n_{9}\right)$. It is depicted in figure 6 .

\section{$5.2 \quad G_{4}$-flux and chiralities}

For the specific base $\mathbb{P}^{3}$, the full SR-ideal of the ambient space of the CY-fourfold $X_{F_{16}}$ reads

$$
\begin{array}{r}
S R=\left\{u e_{4}, u e_{5}, u w, u v, u e_{6}, u e_{3}, e_{1} e_{5}, e_{1} w, e_{1} v, e_{1} e_{6}, e_{1} e_{3}, e_{1} e_{2}, e_{4} w, e_{4} v, e_{4} e_{6},\right. \\
\left.e_{4} e_{3}, e_{4} e_{2}, e_{5} v, e_{5} e_{6}, e_{5} e_{3}, e_{5} e_{2}, w e_{6}, w e_{3}, w e_{2}, v e_{3}, v e_{2}, e_{6} e_{2}, x_{0} x_{1} x_{2} x_{3}\right\} .
\end{array}
$$

Here $[u: v: w]$ and the $e_{i}, i=1 \ldots, 5$ are the homogeneous coordinates on $\mathbb{P}_{F_{16}}$ and $x_{j}$, $j=0, \ldots, 3$, denote homogeneous coordinates on $\mathbb{P}^{3}$. As a basis for $H^{(1,1)}\left(X_{F_{16}}\right)$ we choose

$$
H^{(1,1)}\left(X_{F_{16}}\right)=\left\langle H_{B}, S_{0}, D_{1}^{\mathrm{SU}(3)_{1}}, D_{2}^{\mathrm{SU}(3)_{1}}, D_{1}^{\mathrm{SU}(3)_{2}}, D_{2}^{\mathrm{SU}(3)_{2}}, D_{1}^{\mathrm{SU}(3)_{3}}, D_{2}^{\mathrm{SU}(3)_{3}}\right\rangle .
$$

Next, we compute the vertical cohomology ring of $X_{F_{16}}$ as described in section 2 as a quotient ring using the SR-ideal (5.6) together with the following intersection numbers, that descend from the toric intersections in $\mathbb{P}_{F_{16}}$ :

$$
\begin{aligned}
H_{B}^{3} \cdot S_{0}^{2}=-1, & H_{B}^{3} \cdot\left(D_{2}^{\mathrm{SU}(3)_{1}}\right)^{2}=-2, \\
H_{B}^{3} \cdot\left(D_{2}^{\mathrm{SU}(3)_{2}}\right)^{2}=-2, & H_{B}^{3} \cdot\left(D_{2}^{\mathrm{SU}(3)_{3}}\right)^{2}=-2 .
\end{aligned}
$$

With this information at hand we can obtain all quartic intersections in $X_{F_{16}}$. The dimension of $H_{V}^{(2,2)}\left(X_{F_{16}}\right)$ is found after taking all possible products of two elements in $H^{(1,1)}$ and evaluating the rank of their inner product matrix, yielding

$$
\operatorname{dim}\left(H_{V}^{(2,2)}\left(X_{F_{16}}\right)\right)=9 .
$$


As a basis for $H_{V}^{(2,2)}\left(X_{F_{16}}\right)$ we choose

$$
\begin{gathered}
H_{V}^{(2,2)}\left(X_{F_{16}}\right)=\left\langle H_{B}^{2}, H_{B} S_{0}, D_{1}^{\mathrm{SU}(3)_{1}} H_{B}, D_{2}^{\mathrm{SU}(3)_{1}} H_{B}, D_{1}^{\mathrm{SU}(3)_{2}} H_{B}, D_{2}^{\mathrm{SU}(3)_{2}} H_{B},\right. \\
\left.D_{1}^{\mathrm{SU}(3)_{3}} H_{B}, D_{2}^{\mathrm{SU}(3)_{3}} H_{B}, S_{0}^{2}\right\rangle,
\end{gathered}
$$

which we use to make an ansatz for the most general $G_{4}$-flux. Since the $G_{4}$-flux must be consistent with the matching of M- and F-theoretical CS terms, we have to impose the constraints (2.6), which amount to eight independent constrains. We are left with the following one parameter $G_{4}$-flux:

$$
\begin{aligned}
G_{4}=a_{9}[- & \frac{1}{3} H_{B} \cdot\left(n_{7} D_{1}^{\mathrm{SU}(3)_{3}}+2 n_{7} D_{2}^{\mathrm{SU}(3)_{3}}+n_{9} D_{1}^{\mathrm{SU}(3)_{2}}+2 n_{9} D_{2}^{\mathrm{SU}(3)_{2}}\right) \\
& \left.+n_{7} n_{9} H_{B}^{2}+4 H_{B} \cdot S_{0}+S_{0}^{2}\right] .
\end{aligned}
$$

The parameter $a_{9}$ must be consistently quantized so that the $G_{4}$-flux quantization condition (2.1) with $c_{2}\left(X_{F_{16}}\right)$ as given in (5.4) is obeyed. Again, we ensure the quantization indirectly by choosing $a_{9}$ so that the number $n_{\mathrm{D} 3}$ is a positive integer and that all $3 \mathrm{D}$ CS-terms (2.3) are integral. This issue is discussed below.

As a first step towards the computation of the $4 \mathrm{D}$ matter chiralities we provide the homology classes for the matter surfaces. Using the results from table 8, they read

$$
\begin{aligned}
& \mathcal{C}_{(\mathbf{3}, \overline{\mathbf{3}}, \mathbf{1})}^{w}=\mathcal{S}_{7}\left(3\left[K_{B}^{-1}\right]-\mathcal{S}_{7}-\mathcal{S}_{9}\right)\left(H-E_{1}+\mathcal{S}_{9}\right) \\
& \mathcal{C}_{(\mathbf{3}, \mathbf{1}, \overline{\mathbf{3}})}^{w}=\mathcal{S}_{9}\left(3\left[K_{B}^{-1}\right]-\mathcal{S}_{7}-\mathcal{S}_{9}\right)\left(H-E_{2}+\mathcal{S}_{9}\right) \\
& \left.\mathcal{C}_{(\mathbf{1}, \mathbf{3}, \overline{\mathbf{3}})}^{w}=\mathcal{S}_{7} \mathcal{S}_{9}\left(2 H-E_{1}-E_{2}-E_{3}-E_{4}+K_{B}^{-1}\right]-\mathcal{S}_{7}+\mathcal{S}_{9}\right)
\end{aligned}
$$

where for each matter surface we have chosen a node in the fiber at codimension two with weight $w$ of the respective representation which is not intersected by the zero section. Integrating the $G_{4}$-flux over the surfaces (5.12) leads according to (2.7) the following chiral indices:

$$
\chi_{(\mathbf{3}, \overline{\mathbf{3}}, \mathbf{1})}=\chi_{(\mathbf{3}, \mathbf{1}, \overline{\mathbf{3}})}=\chi_{(\mathbf{1}, \mathbf{3}, \overline{\mathbf{3}})}=\frac{1}{3} n_{7} n_{9}\left(-12+n_{7}+n_{9}\right) a_{9} .
$$

Note that all chiralities are equal, as expected in order for the cubic $\mathrm{SU}(3)$ anomalies to cancel. We also emphasize that at the boundary of the allowed region (see figure 6) we can not have a chiral theory, as all chiralities (5.13) vanish there.

As before, we express the parameter $a_{9}$ in terms of the number of families $b \equiv \chi_{(\mathbf{3}, \overline{\mathbf{3}}, \mathbf{1})}$ as

$$
a_{9}=\frac{3 b}{n_{7} n_{9}\left(-12+n_{7}+n_{9}\right)} .
$$

For all allowed values of $\left(n_{7}, n_{9}\right)$, we explore which positive integral values for $b$ lead to a canceled D3-brane tadpole with a positive integral number $n_{\mathrm{D} 3}$ of D3-branes without adding horizontal $G_{4}$-flux. As shown in table 6, we find that for three families ( $b=3$ ), which is also the minimal value of families, the D3-brane tadpole is canceled at nine different values for 


\begin{tabular}{|c|cccccccccc|}
\hline$n_{7} \backslash^{n_{9}}$ & 1 & 2 & 3 & 4 & 5 & 6 & 7 & 8 & 9 & 10 \\
\hline 10 & $(5 ; 120)$ & & & & & & & & & \\
9 & $(3 ; 94)$ & $(3 ; 94)$ & & & & & & & & \\
8 & $(4 ; 72)$ & $(8 ; 69)$ & $(4 ; 72)$ & & & & & & & \\
7 & $(14 ; 48)$ & $(7 ; 54)$ & $(7 ; 54)$ & $(14 ; 48)$ & & & & & & \\
6 & $(5 ; 50)$ & $(8 ; 44)$ & $(3 ; 44)$ & $(8 ; 44)$ & $(5 ; 50)$ & & & & & \\
5 & $(5 ; 50)$ & $(5 ; 42)$ & $(10 ; 36)$ & $(10 ; 36)$ & $(5 ; 42)$ & $(5 ; 50)$ & & & & \\
4 & $(14 ; 48)$ & $(8 ; 44)$ & $(10 ; 36)$ & $(16 ; 30)$ & $(10 ; 36)$ & $(8 ; 44)$ & $(14 ; 48)$ & & & \\
3 & $(4 ; 72)$ & $(7 ; 54)$ & $(3 ; 44)$ & $(10 ; 36)$ & $(10 ; 36)$ & $(3 ; 44)$ & $(7 ; 54)$ & $(4 ; 72)$ & & \\
2 & $(3 ; 94)$ & $(8 ; 69)$ & $(7 ; 54)$ & $(8 ; 44)$ & $(5 ; 42)$ & $(8 ; 44)$ & $(7 ; 54)$ & $(8 ; 69)$ & $(3 ; 94)$ & \\
1 & $(5 ; 120)$ & $(3 ; 94)$ & $(4 ; 72)$ & $(14 ; 48)$ & $(5 ; 50)$ & $(5 ; 50)$ & $(14 ; 48)$ & $(4 ; 72)$ & $(3 ; 94)$ & $(5 ; 120)$ \\
\hline
\end{tabular}

Table 9. The entries $\left(b ; n_{\mathrm{D} 3}\right)$ show the minimal number of families $b$ for which the number of D3 branes $n_{\mathrm{D} 3}$ is integral and the 3D CS-terms are quantized.

$\left(n_{7}, n_{9}\right)$. We have also checked for the three-family models, that all 3D CS-terms (2.3) are integral. We observe that table 6 is symmetric as expected by the symmetries of polyhedron $F_{16}$ and that there is a family structure for every allowed value of $\left(n_{7}, n_{9}\right)$.

As a cross-check of our results (5.13) we verify the matching (2.5) of CS-terms in Fand M-theory. The non-vanishing CS-terms on the F-theory side computed using (2.4) read

$$
\begin{array}{ll}
\Theta_{11}^{\mathrm{F}}=-\left(\chi_{(\mathbf{3}, \overline{\mathbf{3}}, \mathbf{1})}+\chi_{(\mathbf{3}, \mathbf{1}, \overline{\mathbf{3}})}\right), & \Theta_{12}^{\mathrm{F}}=\frac{1}{2}\left(\chi_{(\mathbf{3}, \overline{\mathbf{3}}, \mathbf{1})}+\chi_{(\mathbf{3}, \mathbf{1}, \overline{\mathbf{3}})}\right), \\
\Theta_{22}^{\mathrm{F}}=-\left(\chi_{(\mathbf{3}, \overline{\mathbf{3}}, \mathbf{1})}+\chi_{(\mathbf{3}, \mathbf{1}, \overline{\mathbf{3}})}\right), & \\
\Theta_{33}^{\mathrm{F}}=-2 \chi_{(\mathbf{1}, \mathbf{3}, \overline{\mathbf{3}})}, & \Theta_{34}^{\mathrm{F}}=\frac{1}{2}\left(3 \chi_{(\mathbf{3}, \overline{\mathbf{3}}, \mathbf{1})}-\chi_{(\mathbf{1}, \mathbf{3}, \overline{\mathbf{3}})}\right), \\
\Theta_{44}^{\mathrm{F}}=-\left(3 \chi_{(\mathbf{3}, \overline{\mathbf{3}}, \mathbf{1})}-\chi_{(\mathbf{1}, \mathbf{3}, \overline{\mathbf{3}})}\right), & \\
\Theta_{55}^{\mathrm{F}}=-\left(3 \chi_{(\mathbf{3}, \overline{\mathbf{3}}, \mathbf{1})}-\chi_{(\mathbf{1}, \mathbf{3}, \overline{\mathbf{3}})}\right), & \Theta_{56}^{\mathrm{F}}=\frac{1}{2}\left(3 \chi_{(\mathbf{3}, \overline{\mathbf{3}}, \mathbf{1})}-\chi_{(\mathbf{1}, \mathbf{3}, \overline{\mathbf{3}})}\right), \\
\Theta_{66}^{\mathrm{F}}=-2 \chi_{(\mathbf{1}, \mathbf{3}, \overline{\mathbf{3}})} . &
\end{array}
$$

We readily compute the CS-terms (2.3) in M-theory, which allows us to reproduce precisely the chiralities in (5.13).

\subsection{Phenomenological discussion}

The breaking from the Trinification model to the SM proceeds via two successive Higgsings. Geometrically, the Higgsings correspond to blow-downs in $X_{F_{16}}$ induced by toric blowdowns in the ambient space $\mathbb{P}_{F_{16}}$ of the elliptic fiber. Thus, we can geometrically visualize the Higgsing directly in the toric diagram of $F_{16}$, see [22] for details.

More concretely, in order to obtain the CY-hypersurface $X_{F_{11}}$ starting from $X_{F_{16}}$, we have to perform two blow-downs in the fiber of $X_{F_{16}}$ that are identified by requiring that the fiber polyhedron $F_{16}$ is mapped to $F_{11}$. There are three possible ways to achieve this. However all of these are equivalent due to the symmetries of the polyhedron. For concreteness we choose here the transition $X_{F_{16}} \rightarrow X_{F_{11}}$ induced by blowing down the 
divisor $e_{5}=0$ and subsequently the divisor $e_{4}=0$ in the toric diagram of $F_{16}$, see figure 5 . Note that after the blow downs we indeed get the toric diagram of $F_{11}$ in figure 1 reflected along the horizontal axis passing through the origin.

The blow-down process restricts the allowed region in figure 6 of $X_{F_{16}}$ due to the requirement that the divisors associated to the sections $s_{3}$ and $s_{5}$, which are part of (3.1) but not of (5.1), are effective. This removes all models which lie above the line $4+n_{7}-n_{9}=0$ in figure 6. Once we exclude these un-Higgsable models, we can compare the number of generations in $X_{F_{16}}$ and $X_{F_{11}}$ in figures 2 and 9. In order to perform such a comparison we have to bear in mind that the polyhedron obtained by Higgsing $X_{F_{16}}$ and the polyhedron specifying $X_{F_{11}}$ are related by a reflection, as mentioned above. Thus, one has to perform a redefinition of the integers $\left(n_{7}, n_{9}\right)$ before and after Higgsing which amounts to the shift $\left(n_{7}, n_{9}\right) \rightarrow\left(8-n_{7}, n_{9}\right)$. Under this map we see that the point $\left(n_{7}, n_{9}\right)=(3,6)$ in $X_{F_{16}}$ maps to $(5,6)$ in $X_{F_{11}}$, both of supporting models with $b=3$. This suggests that there is a toric Higgsing of a Trinification model to a SM with three families. We take this as the example for the following phenomenological discussion.

On the field theory side the described transition $X_{F_{16}} \rightarrow X_{F_{11}}$ proceeds by a VEV of the field in the representation $(\mathbf{3}, \overline{\mathbf{3}}, \mathbf{1})$ at the matter curve $s_{1}=s_{7}=0$, cf. table 8 . For reasons that will become clear in the following, the Higgsing down to the MSSM is only possible if in addition to the three chiral families one also has two vector-like pairs $(\mathbf{3}, \overline{\mathbf{3}}, \mathbf{1})$, $(\overline{\mathbf{3}}, \mathbf{3}, \mathbf{1})$ [107]. While one pair, which we denote as $H^{1}, \bar{H}^{1}$ is needed for the intermediate breaking $\mathrm{SU}(3)^{3} / \mathbb{Z}_{3} \rightarrow \mathrm{SU}(3) \times \mathrm{SU}(2)^{2} \times \mathrm{U}(1)$, the second pair $H^{2}, \bar{H}^{2}$ is needed because the representation, which breaks the $\mathrm{SU}(2) \times \mathrm{U}(1)$ down to the hypercharge generator, is contained in the $(\mathbf{3}, \overline{\mathbf{3}}, \mathbf{1})$ representation, too. Therefore, in addition to the three generations of chiral fields $\left(\lambda_{i}, \mathcal{Q}_{i}, \overline{\mathcal{Q}}_{i}\right)$, we assume that our model allows for two massless vector-like pairs of Higgs fields $H^{1}, \bar{H}^{1}$ and $H^{2}, \bar{H}^{2}$.

In the first Higgsing inducing the symmetry breaking $\mathrm{SU}(3)^{3} / \mathbb{Z}_{3} \rightarrow \mathrm{SU}(3) \times \mathrm{SU}(2)^{2} \times$ $\mathrm{U}(1)$ we arrive at the following decomposition of fields composing the chiral families of our model:

$$
\begin{aligned}
\lambda_{i} & =(\mathbf{3}, \overline{\mathbf{3}}, \mathbf{1}) \rightarrow(\mathbf{2}, \mathbf{2}, \mathbf{1})_{0}+(\mathbf{1}, \mathbf{2}, \mathbf{1})_{-3}+(\mathbf{2}, \mathbf{1}, \mathbf{1})_{3}+(\mathbf{1}, \mathbf{1}, \mathbf{1})_{0} \\
\mathcal{Q}_{i} & =(\overline{\mathbf{3}}, \mathbf{1}, \mathbf{3}) \rightarrow(\mathbf{2}, \mathbf{1}, \mathbf{3})_{-1}+(\mathbf{1}, \mathbf{1}, \mathbf{3})_{+2} \\
\overline{\mathcal{Q}}_{i} & =(\mathbf{1}, \mathbf{3}, \overline{\mathbf{3}}) \rightarrow(\mathbf{1}, \mathbf{2}, \overline{\mathbf{3}})_{1}+(\mathbf{1}, \mathbf{1}, \overline{\mathbf{3}})_{-2}
\end{aligned}
$$

Similarly the Higgses of the Trinification model decompose as

$$
\begin{aligned}
& H^{1}, H^{2}=(\mathbf{3}, \overline{\mathbf{3}}, \mathbf{1}) \rightarrow(\mathbf{2}, \mathbf{2}, \mathbf{1})_{0}+(\mathbf{1}, \mathbf{2}, \mathbf{1})_{-3}+(\mathbf{2}, \mathbf{1}, \mathbf{1})_{+3}+(\mathbf{1}, \mathbf{1}, \mathbf{1})_{0}, \\
& \bar{H}^{1}, \bar{H}^{2}=(\overline{\mathbf{3}}, \mathbf{3}, \mathbf{1}) \rightarrow(\mathbf{2}, \mathbf{2}, \mathbf{1})_{0}+(\mathbf{1}, \mathbf{2}, \mathbf{1})_{+3}+(\mathbf{2}, \mathbf{1}, \mathbf{1})_{-3}+(\mathbf{1}, \mathbf{1}, \mathbf{1})_{0} .
\end{aligned}
$$

Here, the generator of the unbroken $\mathrm{U}(1)$ is given by

$$
Q=T_{1}^{8}+T_{2}^{8}, \quad \text { with } \quad T_{1,2}^{8}=\operatorname{diag}(1,1,-2),
$$

where the subscripts 1, 2 label the corresponding $\mathrm{SU}(3)$ factors.

Next we need to break one of the $\mathrm{SU}(2)$ factors together with the $\mathrm{U}(1)$-factor to the hypercharge $\mathrm{U}(1)_{Y}$. To this end, one of the fields in either the representation $(\mathbf{1}, \mathbf{2}, \mathbf{1})_{-3}$ 


\begin{tabular}{|ccl|}
\hline Tri-Rep & SM decomposition \\
\hline$\lambda_{i}$ & $\rightarrow$ & $H_{d, i}:(\mathbf{1}, \mathbf{2})_{-1 / 2}, H_{u, i}:(\mathbf{1}, \mathbf{2})_{1 / 2}, \bar{e}_{i}:(\mathbf{1}, \mathbf{1})_{1}$, \\
& & $L_{i}:(\mathbf{1}, \mathbf{2})_{-1 / 2}, \bar{\nu}_{i}^{1}:(\mathbf{1}, \mathbf{1})_{0}, \bar{\nu}_{i}^{2}:(\mathbf{1}, \mathbf{1})_{0}$ \\
$\mathcal{Q}_{i}$ & $\rightarrow$ & $Q_{i}:(\mathbf{3}, \mathbf{2})_{\frac{1}{6}}, D_{i}:(\overline{\mathbf{3}}, \mathbf{1})_{-\frac{1}{3}}$ \\
$\overline{\mathcal{Q}}_{i}$ & $\rightarrow$ & $\bar{u}:(\overline{\mathbf{3}}, \mathbf{1})_{-\frac{2}{3}}, \bar{d}_{i}:(\overline{\mathbf{3}}, \mathbf{1})_{\frac{1}{3}}, \bar{D}_{i}:(\overline{\mathbf{3}}, \mathbf{1})_{\frac{1}{3}}$ \\
\hline$H^{1}, \bar{H}^{1} \rightarrow$ & $H_{d}^{1}, \bar{H}_{u}^{1}:(\mathbf{1}, \mathbf{2})_{-1 / 2}, H_{u}^{1}, \bar{H}_{d}^{1}:(\mathbf{1}, \mathbf{2})_{1 / 2}$ \\
$H^{2}, \bar{H}^{2} \rightarrow$ & $H_{d}^{2}, \bar{H}_{u}^{2}, H_{d}^{2 \prime}:(\mathbf{1}, \mathbf{2})_{-1 / 2}, H_{u}^{1}, \bar{H}_{d}^{1}, H_{d}^{2 \prime}:(\mathbf{1}, \mathbf{2})_{1 / 2}$ \\
\hline
\end{tabular}

Table 10. The Trinification representations decomposed in terms of their MSSM constituents. We have also included the charged fields inside of the fields $H^{1}, H^{2}$ which do not participate in the Higgs process.

\begin{tabular}{|c|c|}
\hline Yukawa & Locus \\
\hline$(\mathbf{3}, \overline{\mathbf{3}}, \mathbf{1}) \cdot \overline{(\mathbf{3}, \mathbf{1}, \overline{\mathbf{3}}) \cdot(\mathbf{1}, \mathbf{3}, \overline{\mathbf{3}})}$ & $s_{1}=s_{7}=s_{9}=0$ \\
\hline$(\mathbf{3}, \overline{\mathbf{3}}, \mathbf{1}) \cdot(\mathbf{3}, \overline{\mathbf{3}}, \mathbf{1}) \cdot(\mathbf{3}, \overline{\mathbf{3}}, \mathbf{1})$ & $s_{1}=s_{6}=s_{7}=0$ \\
\hline$(\mathbf{3}, \mathbf{1}, \overline{\mathbf{3}}) \cdot(\mathbf{3}, \mathbf{1}, \overline{\mathbf{3}}) \cdot(\mathbf{3}, \mathbf{1}, \overline{\mathbf{3}})$ & $s_{1}=s_{6}=s_{8}=0$ \\
\hline$(\mathbf{1}, \mathbf{3}, \overline{\mathbf{3}}) \cdot(\mathbf{1}, \mathbf{3}, \overline{\mathbf{3}}) \cdot(\mathbf{1}, \mathbf{3}, \overline{\mathbf{3}})$ & $s_{6}=s_{7}=s_{9}=0$ \\
\hline
\end{tabular}

Table 11. Codimension three loci and corresponding Yukawa points for $X_{F_{16}}$.

or $(\mathbf{2}, \mathbf{1}, \mathbf{1})_{+3}$ in the decomposition of the representation $(\mathbf{3}, \overline{\mathbf{3}}, \mathbf{1})$ has to pick a VEV. Note also that for the pair $H^{1}, \bar{H}^{1}$, both of these fields (and their complex conjugates) will be absorbed as the longitudinal modes of the massive vector fields. Hence, we have to pick the fields for the second Higgsing as irreducible representations stemming from the decomposition of $H^{2}$ and $\bar{H}^{2}$ in (5.17). For concreteness we take the states $(\mathbf{1}, \mathbf{2}, \mathbf{1})_{-3} \subset H^{2}$, $(\mathbf{1}, \mathbf{2}, \mathbf{1})_{+3} \subset \bar{H}^{2}$ to be responsible for the second breaking. In that case the hypercharge generator written in terms of the $\mathrm{U}(1)$ generator $Q$ and the Cartan generator $T^{3}$ of the first $\mathrm{SU}(2)$ reads

$$
Q_{Y}=-\left(T^{3}+Q / 6\right)
$$

The decomposition of the chiral matter representations in terms of the SM gauge group is given in table 10. Here we immediately see that each family at the Trinification level provides an entire SM family, extended by two right handed neutrinos $\bar{\nu}_{1, i}, \bar{\nu}_{2, i}$, a vectorlike pair of color triplets $D_{i}, \bar{D}_{i}$ and a pair of SM-like Higgs fields $H_{u, i}, H_{d, j}$. For the decoupling of the exotics and the discussion of the couplings of the fields, we have to refer to the geometrically allowed Yukawa couplings in $X_{F_{16}}$ which are given in table 11 [22]. First notice that in general one has two scales $\Lambda_{1}$ and $\Lambda_{2}$ associated to each of the two symmetry breakings from Trinification to SM. However, radiative corrections will push these scales towards each other, and due to that we can simply assume that both Higgsings occur simultaneously at some scale $\Lambda$. Given the allowed couplings $H^{1} \mathcal{Q}_{i} \overline{\mathcal{Q}}_{i}$ and $H^{2} \mathcal{Q}_{i} \overline{\mathcal{Q}}_{j}$ 
we see that the exotic triplets $D_{i}, \bar{D}_{i}$ pick up a mass of order $\Lambda$. We also note from table 10 that we get eight pairs of SM-like Higgses. Nevertheless, working out all bilinears among them, which result from the three point couplings of the Trinification model with VEV insertions, one sees that essentially all of the Higgses get lifted at the Trinification scale $\Lambda$. One might hope that this can be fixed by tuning the complex structure of $X_{F_{16}}$ to suppress the corresponding Yukawa couplings, however, we have to recall that there are only two structurally different types of Yukawa couplings. Indeed, on the one hand we have a three point coupling involving a single matter curve. On the other hand we have a coupling involving the three different matter curves, which if suppressed, will imply that Yukawa couplings for quarks and leptons are suppressed.

We conclude with another remark regarding the presence of vector-like pairs in the model. As we discussed above, we need vector-like matter $H_{1}, \bar{H}_{1}, H_{2}$ and $\bar{H}_{2}$ in the representations $(\overline{\mathbf{3}}, \mathbf{3}, \mathbf{1})$ and $(\mathbf{3}, \overline{\mathbf{3}}, \mathbf{1})$, respectively, for the Higgsing down to the MSSM. It is then natural to expect also additional vector-like matter in the representations $(\overline{\mathbf{3}}, \mathbf{1}, \mathbf{3})$ and $(\mathbf{1}, \mathbf{3}, \overline{\mathbf{3}})$, which would give rise to exotics beyond those presented in table $10 .{ }^{16}$ Indeed, this can be motivated geometrically by the $\mathbb{Z}_{3}$-symmetry of the polyhedron $F_{16}$. We have seen that this symmetry is realized on the chiral spectrum in table 9, relating different theories that only differ by a permutation of their $\mathrm{SU}(3)$ factors. We expect this to hold more generally for the vector-like sectors of different theories related by the $\mathbb{Z}_{3}$ symmetry. In particular for the stratum $n_{7}=n_{9}=4$, which is a fixed point of that a symmetry, one expects the same number of vector-like pairs for all representations. For these type of models one expects unification of the $\mathrm{SU}(3)$ gauge couplings above the Trinification scale [108]. Furthermore, in $[107,109]$ the possibility of having a light SM Higgs pair has been related to the presence of additional discrete $R$ and non- $R$ symmetries in Trinification models with vector-like pairs. These symmetries could reduce to the standard matter parity after the Trinification group is broken down to the SM gauge group, and hence they could prevent the model from an exceedingly fast proton decay. It would be interesting to find ways to realize these additional symmetries in F-theory.

\section{Conclusions}

In this work we have presented explicit, globally consistent four-dimensional F-theory compactifications that have the standard model gauge group and three chiral families. Moreover we considered embeddings of the standard model into the Pati-Salam or trinification models using a toric realization of the relevant Higgs effects.

The models considered in this work result from F-theory compactifications on CalabiYau fourfolds, with their elliptic fibers defined as toric hypersurfaces in the three 2D toric ambient spaces $\mathbb{P}_{F_{11}}, \mathbb{P}_{F_{13}}$ and $\mathbb{P}_{F_{16}}$, see e.g. [22]. While the gauge symmetry, the type of matter representations and the possible Yukawa couplings are properties independent of the choice for the base manifold, we fix the base manifold to be $B=\mathbb{P}^{3}$ in order to construct the vertical cohomology in each case. This allows us to find explicit solutions for the chirality-inducing $G_{4}$-flux and ultimately, to determine the possible $4 \mathrm{D}$ chiralities of

\footnotetext{
${ }^{16}$ Again, it is expected that these exotics acquire masses of order $\Lambda$, too.
} 
matter fields. The expressions for the $G_{4}$-flux in each type of compactification are shown to satisfy the consistency conditions imposed by the matching of F- and M-theoretical Chern-Simons terms, as well a the D3-brane tadpole cancelation condition with a positive, integral number of D3-branes. The $G_{4}$-flux quantization condition is ensured indirectly by the analogous quantization condition of the 3D CS-terms. We also show that the obtained chiralities are consistent with field theoretic calculations of CS-terms and anomalies.

In this work we have only considered the vertical part of the middle cohomology, which is responsible for the generation of chirality. With no $G_{4}$-flux along the horizontal components we observe that the D3-brane tadpole cancellation imposes very stringent constraints on the minimal number of generations permitted in each of the models, which happens to be precisely three. We explicitly identify the consistent three-family models in our list of concrete models. Although addition of horizontal $G_{4}$-flux $G_{4}^{H}$ does not directly change the chiralities, the divisibility properties of $\chi\left(X_{4}\right) / 24-1 / 2\left(G_{4}^{H}\right)^{2}$ might be improved compared to that of $\chi\left(X_{4}\right) / 24$ alone, yielding weaker constraints on the number of families imposed by the integrality of $n_{D_{3}} .{ }^{17}$ Thus, we expect a greater number of consistent three-family models.

For the Pati-Salam and Trinification models we also described the Higgs transition to the Standard Model. On the geometric side, effectiveness conditions of the divisor classes needed to specify the models after the transition determine points in the allowed region for $\left(n_{7}, n_{9}\right)$ of $X_{F_{13}}$ and $X_{F_{16}}$, for which the Higgsing is possible. While in the six-dimensional case, these conditions are known to ensure a D-flat Higgsing, in four dimensions, a similar field theoretical understanding remains elusive and requires a better understanding of vector-like matter in F-theory, beyond chiral indices. Indeed, we presume that for values of $\left(n_{7}, n_{9}\right)$, where some divisors fail to be effective, one does not have a light pair of vector-like fields to carry out the Higgsing. Due to the current lack of control of the vector-like sector of F-theory, we simply assume that over the points where all divisors defining the model remain effective after the transition, one has the necessary vector-like pairs of Higgses and that the transition is indeed possible. The verification of this assumption is left for future works. Since the supersymmetric Higgs mechanism does not change the net chiralities, we expect that a model based on $X_{F_{13}}$ or $X_{F_{16}}$ with three families at a given point $\left(n_{7}, n_{9}\right)$ maps to a model with three families in $X_{F_{11}}$, at the same point $\left(n_{7}, n_{9}\right)$. Remarkably for both $X_{F_{13}}$ and $X_{F_{16}}$ we find a point with three families before and after Higgsing. If we in addition require that the number of D3-branes remains constant in the transition, we must add horizontal $G_{4}$-flux after the Higgsing in order to compensate for the change in the Euler number of the CY-fourfold. The systematic inclusion of horizontal $G_{4}$-flux and their effect on the Higgsing, as well as the connection to $G_{4}$-flux quantization, would be interesting to study in future works.

In this work we have also made some remarks on the phenomenology of the studied models. A rough look at the superpotential at quadratic and cubic order of the resulting effective field theories lead to the well known observation that the bare models have some

\footnotetext{
${ }^{17}$ There might be a finer integral basis of $H^{4}\left(X_{4}\right)$ that is off-diagonal in the splitting into horizontal and vertical cohomology.
} 
phenomenologically unappealing features, such as the prediction of a fast proton decay or the difficulty to retain a light pair of electroweak Higgs fields in the spectrum. However, it is likely that the proton decay operators can be kept under control if one goes to special points in complex structure moduli space of the CY-fourfolds. On the field theory side this usually corresponds to the existence of an accidental discrete symmetry. It would be very interesting whether there exists horizontal $G_{4}$-flux that stabilizes the complex structure of the CY-fourfold at these points in moduli, following the general arguments discussed in $[86]$.

Finally, we have also computed the Hodge numbers of the considered CY-fourfolds with three-families. For our simple choice of base $\mathbb{P}^{3}$ we always have $h^{(2,1)}=0$ which constraints cosmological F-theory applications of our models. Clearly, it is an interesting future direction to extend the phenomenological analysis carried out in this work to other bases $B$ allowing for richer possible applications to cosmology.

\section{Acknowledgments}

We would like to thank Hans Jockers, Albrecht Klemm and Hernan Piragua for helpful discussions and comments. M.C. and D.K. would like to thank Paul Langacker for discussions and collaboration on related topics. D.K. thanks the Bethe Center for Theoretical Physics Bonn for hospitality during completion of the project. D.M. thanks the DESY theory group, where part of this work was completed, for hospitality. The work of M.C. is supported by the DOE grant DE-SC0007901, the Fay R. and Eugene L. Langberg Endowed Chairand the Slovenian Research Agency (ARRS). The work of D.M. was partially supported by the Deutsche Forschungsgemeinschaft under the Collaborative Research Center (SFB) 676 Particles, Strings and the Early Universe. The work of P.O. and J.R. is partially supported by a scholarship of the Bonn-Cologne Graduate School BCGS, the SFB-Transregio TR33 The Dark Universe (Deutsche Forschungsgemeinschaft) and the European Union 7th network program Unification in the LHC era (PITN-GA-2009-237920).

\section{A Hodge numbers of CY-fourfolds}

In this appendix we discuss the computation of the Hodge numbers of CY-fourfolds given as toric hypersurfaces. We readily use these methods for the calculation of the Hodge numbers of the three families of CY-fourfolds $X_{F_{11}}, X_{F_{13}}$ and $X_{F_{16}}$.

A CY-fourfold has the independent Hodge numbers $h^{(1,1)}, h^{(2,1)}$ and $h^{(3,1)}$. They are related to the Euler number as

$$
\chi(X)=6\left(8+h^{(1,1)}+h^{(3,1)}-h^{(2,1)}\right) .
$$

Since one can compute $\chi(X)$ independently by integrating the top Chern class $c_{4}(X)$ over $X$, it is sufficient to calculate $h^{(2,1)}$ and $h^{(3,1)}$ to obtain all independent Hodge numbers.

For a CY-fourfold given as a toric hypersurface specified by a pair of dual five dimensional reflexive lattice polyhedra $\left(\Delta, \Delta^{*}\right)$, one can use the combinatorial Batyrev formulas 
(see [76] and references therein) to calculate the Hodge numbers as

$$
\begin{aligned}
h^{(1,1)} & =l(\Delta)-(4+2)-\sum_{\operatorname{dim} \Theta=4} l^{\prime}(\Theta)+\sum_{\operatorname{codim} \Theta_{i}=2} l^{\prime}\left(\Theta_{i}\right) l^{\prime}\left(\Theta_{i}^{*}\right), \\
h^{(3,1)} & =l\left(\Delta^{*}\right)-(4+2)-\sum_{\operatorname{dim} \Theta^{*}=4} l^{\prime}\left(\Theta^{*}\right)+\sum_{\operatorname{codim} \Theta_{i}=2} l^{\prime}\left(\Theta_{i}\right) l^{\prime}\left(\Theta_{i}^{*}\right), \\
h^{(2,1)} & =\sum_{\operatorname{codim} \Theta_{i}=3} l^{\prime}\left(\Theta_{i}\right) l^{\prime}\left(\Theta_{i}^{*}\right) .
\end{aligned}
$$

Here, $\Theta\left(\Theta^{*}\right)$ denote faces of $\Delta\left(\Delta^{*}\right)$, while the sum is over pairs $\left(\Theta_{i}, \Theta_{i}^{*}\right)$ of dual faces. The $l(\Theta)$ and $l^{\prime}(\Theta)$ count the total number of integral points of a face $\Theta$ and the number inside the face $\Theta$, respectively. Finally, $l(\Delta)$ is the total number of integral points of the polyhedron $\Delta$.

In the following we are considering fourfolds that have the elliptic curves in $\mathbb{P}_{F_{11}}, \mathbb{P}_{F_{13}}$ and $\mathbb{P}_{F_{16}}$ as a fiber over a $\mathbb{P}^{3}$ base space. An explicit expression for the total polyhedra of the 5D toric ambient space of these fibrations can be found in appendix B. We only need some general observations about these polyhedra here. The polytope of the $\mathbb{P}^{3}$ base always contributes four points. Hence we can write

$$
l(\Delta)=4+\# \operatorname{Points}\left(F_{i}\right) .
$$

For all fibrations of this type we find that there are never points within codimension two and three facets of the polyhedra i.e.

$$
l^{\prime}\left(\Theta_{\text {codim }=2, i}\right)=0 \quad(\forall i), \quad l^{\prime}\left(\Theta_{\text {codim }=3, i}\right)=0 \quad(\forall i) .
$$

Furthermore we use the following observation that we made in [22], namely

$$
\text { \#Points }\left(F_{i}\right)-4=\operatorname{Rank}\left(G_{F_{i}}\right) \text {, }
$$

at a generic stratum of the fibration, i.e. on a bulk stratum of the allowed region. Hence the above formula (A.2) for the Hodge number $h^{(1,1)}$ simplifies to

$$
h^{(1,1)}=\# \operatorname{Points}\left(F_{i}\right)-2-\sum_{\operatorname{dim} \Theta=4} l^{\prime}(\Theta)=\operatorname{Rank}\left(G_{F_{i}}\right)+2 .
$$

Note that, in contrast to this formula, the formula (A.2) is valid for all strata of the fibration even at the boundary of the allowed regions were divisors are switched off and the rank of the gauge group is reduced. This rank reduction is precisely taken care of by vertices that move into the interior of dimension four facets and therefore correct the above formula for the $h^{(1,1)}$ numbers.

Similarly we find, that for all of our models

$$
h^{(2,1)}=0 .
$$

Using (A.1) we can thus give a closed formula for all $h^{(3,1)}$

$$
h^{(3,1)}=\frac{\chi}{6}-10-\operatorname{Rank}\left(G_{F_{i}}\right) .
$$




\begin{tabular}{|c|ccc|cc|}
\hline variable & \multicolumn{2}{|c|}{ Base Vertex } & \multicolumn{2}{c|}{ Fiber Vertex } \\
\hline$z_{1}$ & 1 & 1 & 1 & $n_{9}-4$ & $4-n_{7}$ \\
$z_{2}$ & -1 & 0 & 0 & 0 & 0 \\
$z_{4}$ & 0 & -1 & 0 & 0 & 0 \\
$z_{3}$ & 0 & 0 & -1 & 0 & 0 \\
\hline$\hat{v}_{0}$ & 0 & 0 & 0 & 0 & 0 \\
$\hat{v}_{i}$ & 0 & 0 & 0 & \multicolumn{2}{|c|}{$v_{i}$} \\
\hline
\end{tabular}

Table 12. The five dimensional polyhedron describing a $\mathbb{P}^{3}$ fibered fourfold with the two dimensional fiber coordinates $v_{i}$. The first four coordinates describe the $\mathbb{P}^{3}$ and the choice of $n_{7}$ and $n_{9}$ fix the fibration.

Furthermore, by having fixed the above Hodge numbers we can obtain the Hodge number $h^{(2,2)}$ as well using

$$
h^{(2,2)}=2\left(22+2 h^{(1,1)}+2 h^{(3,1)}-h^{(2,1)}\right)=2\left(6+\frac{\chi}{3}\right) .
$$

By using the explicit presentation of the vertical cohomology ring $H_{V}^{(2,2)}\left(X_{F_{i}}\right)$ as a quotient ring, we can compute $h_{V}^{(2,2)}$. With the knowledge of the full $h^{(2,2)}$ we can compute the dimension of the horizontal cohomology.

We note that the absence of any $(2,1)$-forms is of particular interest for cosmological applications as the resulting three-forms could be used to obtain axions to drive inflation as discussed in an F-theory context in $[110,111]$. However these specific type of axions are absent in all of our models. We conclude that the $\mathbb{P}^{3}$ base is too simple to allow for these features. ${ }^{18}$

\section{B Concrete toric lattice polyhedra of $4 \mathrm{D}$ chiral models}

Using the algorithm of [58] we can construct the CY-manifolds $X_{F_{i}}$ as concrete toric hypersurfaces associated to a five dimensional lattice polyhedron that specifies the underlying $5 \mathrm{D}$ ambient space. In our case the ambient space is a $\mathbb{P}_{F_{i}}$-fibration with a $\mathbb{P}^{3}$ base specified by two numbers $n_{7}$ and $n_{9}$. The polyhedron is given in table 12 . From the above polyhedron one can deduce dimension one, two, three and four facets and the points within them. From that information we can calculate the amount of Hodge numbers and in particular we find the vanishing of $h^{(2,1)}$ numbers for all $n_{7}$ and $n_{9}$. In the table 13 we specify the Euler and Hodge numbers for the specific fibrations that allow for three-family $G_{4}$-flux. We see that only a transition between loci is possible when it is possible for the Euler number to increase.

\footnotetext{
${ }^{18}$ We still find generically a lot of complex structure moduli $h^{(3,1)}$ that can be used as well. However the specific scenario in [110] is excluded.
} 


\begin{tabular}{|cc|c|c|c|c|c|c|}
\hline Fiber $F_{i}$ & Stratum $\left(n_{7}, n_{9}\right)$ & $\chi$ & $h^{(3,1)}$ & $h^{(1,1)}$ & $h^{(2,1)}$ & $h_{v}^{(2,2)}$ & $h^{2,2}$ \\
\hline$F_{11}$ & $(5,6)$ & 1134 & 175 & 6 & 0 & 7 & 768 \\
$F_{11}$ & $(2,5)$ & 1554 & 245 & 6 & 0 & 7 & 1048 \\
\hline$F_{13}$ & $(5,6)$ & 1080 & 165 & 7 & 0 & 8 & 732 \\
\hline$F_{16}$ & $(3,6)$ & 1062 & 161 & 8 & 0 & 9 & 720 \\
$F_{16}$ & $(2,9)$ & 2274 & 363 & 8 & 0 & 9 & 820 \\
\hline
\end{tabular}

Table 13. The Euler number and Hodge numbers for all inequivalent strata that support three family fluxes.

Open Access. This article is distributed under the terms of the Creative Commons Attribution License (CC-BY 4.0), which permits any use, distribution and reproduction in any medium, provided the original author(s) and source are credited.

\section{References}

[1] C. Vafa, Evidence for F-theory, Nucl. Phys. B 469 (1996) 403 [hep-th/9602022] [InSPIRE].

[2] R. Donagi and M. Wijnholt, Model building with F-theory, Adv. Theor. Math. Phys. 15 (2011) 1237 [arXiv: 0802.2969] [INSPIRE].

[3] C. Beasley, J.J. Heckman and C. Vafa, GUTs and exceptional branes in F-theory - I, JHEP 01 (2009) 058 [arXiv:0802.3391] [INSPIRE].

[4] C. Beasley, J.J. Heckman and C. Vafa, GUTs and exceptional branes in F-theory - II: experimental predictions, JHEP 01 (2009) 059 [arXiv:0806.0102] [INSPIRE].

[5] R. Donagi and M. Wijnholt, Breaking GUT Groups in F-theory, Adv. Theor. Math. Phys. 15 (2011) 1523 [arXiv: 0808.2223] [INSPIRE].

[6] J. Marsano, N. Saulina and S. Schäfer-Nameki, F-theory compactifications for supersymmetric GUTs, JHEP 08 (2009) 030 [arXiv:0904.3932] [INSPIRE].

[7] R. Blumenhagen, T.W. Grimm, B. Jurke and T. Weigand, Global F-theory GUTs, Nucl. Phys. B 829 (2010) 325 [arXiv: 0908.1784] [INSPIRE].

[8] T.W. Grimm, S. Krause and T. Weigand, F-theory GUT vacua on compact Calabi-Yau fourfolds, JHEP 07 (2010) 037 [arXiv:0912.3524] [INSPIRE].

[9] E. Dudas and E. Palti, Froggatt-Nielsen models from $E_{8}$ in F-theory GUTs, JHEP 01 (2010) 127 [arXiv: 0912.0853] [inSPIRE].

[10] J. Marsano, N. Saulina and S. Schäfer-Nameki, Compact F-theory GUTs with U(1)(PQ), JHEP 04 (2010) 095 [arXiv: 0912.0272] [INSPIRE].

[11] E. Dudas and E. Palti, On hypercharge flux and exotics in F-theory GUTs, JHEP 09 (2010) 013 [arXiv: 1007.1297] [INSPIRE].

[12] M.J. Dolan, J. Marsano, N. Saulina and S. Schäfer-Nameki, F-theory GUTs with U(1) symmetries: generalities and survey, Phys. Rev. D 84 (2011) 066008 [arXiv:1102.0290] [INSPIRE]. 
[13] C. Mayrhofer, E. Palti and T. Weigand, U(1) symmetries in F-theory GUTs with multiple sections, JHEP 03 (2013) 098 [arXiv:1211.6742] [INSPIRE].

[14] V. Braun, T.W. Grimm and J. Keitel, New global F-theory GUTs with U(1) symmetries, JHEP 09 (2013) 154 [arXiv:1302.1854] [INSPIRE].

[15] M. Cvetič, A. Grassi, D. Klevers and H. Piragua, Chiral four-dimensional F-theory compactifications with $\mathrm{SU}(5)$ and multiple U(1)-factors, JHEP 04 (2014) 010 [arXiv: 1306.3987] [INSPIRE].

[16] S. Krippendorf, D.K. Mayorga Pena, P.-K. Oehlmann and F. Ruehle, Rational F-theory GUTs without exotics, JHEP 07 (2014) 013 [arXiv: 1401.5084] [INSPIRE].

[17] R. Blumenhagen, Gauge coupling unification in F-theory grand unified theories, Phys. Rev. Lett. 102 (2009) 071601 [arXiv: 0812.0248] [INSPIRE].

[18] M.J. Dolan, J. Marsano and S. Schäfer-Nameki, Unification and Phenomenology of F-theory GUTs with $\mathrm{U}(1)(P Q)$, JHEP 12 (2011) 032 [arXiv:1109.4958] [INSPIRE].

[19] K.-S. Choi and T. Kobayashi, Towards the MSSM from F-theory, Phys. Lett. B 693 (2010) 330 [arXiv:1003.2126] [INSPIRE].

[20] K.-S. Choi, On the standard model group in F-theory, Eur. Phys. J. C 74 (2014) 2939 [arXiv: 1309.7297] [INSPIRE].

[21] L. Lin and T. Weigand, Towards the standard model in F-theory, Fortsch. Phys. 63 (2015) 55 [arXiv: 1406.6071] [INSPIRE].

[22] D. Klevers, D.K. Mayorga Pena, P.-K. Oehlmann, H. Piragua and J. Reuter, F-theory on all toric hypersurface fibrations and its Higgs branches, JHEP 01 (2015) 142 [arXiv: 1408.4808] [INSPIRE].

[23] A. Grassi, J. Halverson, J. Shaneson and W. Taylor, Non-higgsable QCD and the standard model spectrum in F-theory, JHEP 01 (2015) 086 [arXiv:1409.8295] [INSPIRE].

[24] M. Cvetič, T. Li and T. Liu, Supersymmetric Pati-Salam models from intersecting D6-branes: a road to the standard model, Nucl. Phys. B 698 (2004) 163 [hep-th/0403061] [INSPIRE].

[25] S. Förste, H.P. Nilles, P.K.S. Vaudrevange and A. Wingerter, Heterotic brane world, Phys. Rev. D 70 (2004) 106008 [hep-th/0406208] [INSPIRE].

[26] T. Kobayashi, S. Raby and R.-J. Zhang, Searching for realistic $4 D$ string models with a Pati-Salam symmetry: orbifold grand unified theories from heterotic string compactification on a Z(6) orbifold, Nucl. Phys. B 704 (2005) 3 [hep-ph/0409098] [INSPIRE].

[27] T. Weigand, Lectures on F-theory compactifications and model building, Class. Quant. Grav. 27 (2010) 214004 [arXiv: 1009. 3497] [INSPIRE].

[28] A. Maharana and E. Palti, Models of particle physics from type IIB string theory and F-theory: a review, Int. J. Mod. Phys. A 28 (2013) 1330005 [arXiv:1212.0555] [inSPIRE].

[29] D.R. Morrison and C. Vafa, Compactifications of F-theory on Calabi-Yau threefolds. 1, Nucl. Phys. B 473 (1996) 74 [hep-th/9602114] [INSPIRE].

[30] D.R. Morrison and C. Vafa, Compactifications of F-theory on Calabi-Yau threefolds. 2, Nucl. Phys. B 476 (1996) 437 [hep-th/9603161] [INSPIRE]. 
[31] M. Bershadsky, K.A. Intriligator, S. Kachru, D.R. Morrison, V. Sadov and C. Vafa, Geometric singularities and enhanced gauge symmetries, Nucl. Phys. B 481 (1996) 215 [hep-th/9605200] [INSPIRE].

[32] S. Katz, D.R. Morrison, S. Schäfer-Nameki and J. Sully, Tate's algorithm and F-theory, JHEP 08 (2011) 094 [arXiv:1106.3854] [INSPIRE].

[33] P.S. Aspinwall and D.R. Morrison, Nonsimply connected gauge groups and rational points on elliptic curves, JHEP 07 (1998) 012 [hep-th/9805206] [INSPIRE].

[34] P.S. Aspinwall, S.H. Katz and D.R. Morrison, Lie groups, Calabi-Yau threefolds and F-theory, Adv. Theor. Math. Phys. 4 (2000) 95 [hep-th/0002012] [InSPIRE].

[35] S.H. Katz and C. Vafa, Matter from geometry, Nucl. Phys. B 497 (1997) 146 [hep-th/9606086] [INSPIRE].

[36] D.R. Morrison and W. Taylor, Matter and singularities, JHEP 01 (2012) 022 [arXiv:1106.3563] [INSPIRE].

[37] J. Marsano and S. Schäfer-Nameki, Yukawas, G-flux and spectral covers from resolved Calabi-Yau's, JHEP 11 (2011) 098 [arXiv:1108.1794] [INSPIRE].

[38] S. Krause, C. Mayrhofer and T. Weigand, Gauge fluxes in F-theory and type IIB orientifolds, JHEP 08 (2012) 119 [arXiv:1202.3138] [INSPIRE].

[39] D.R. Morrison and W. Taylor, Classifying bases for $6 D$ F-theory models, Central Eur. J. Phys. 10 (2012) 1072 [arXiv:1201.1943] [InSPIRE].

[40] D.R. Morrison and W. Taylor, Toric bases for 6D F-theory models, Fortsch. Phys. 60 (2012) 1187 [arXiv: 1204.0283] [INSPIRE].

[41] G. Martini and W. Taylor, 6D F-theory models and elliptically fibered Calabi-Yau threefolds over semi-toric base surfaces, JHEP 06 (2015) 061 [arXiv: 1404.6300] [INSPIRE].

[42] J.J. Heckman, D.R. Morrison and C. Vafa, On the classification of $6 D$ SCFTs and generalized ADE orbifolds, JHEP 05 (2014) 028 [Erratum ibid. 1506 (2015) 017] [arXiv: 1312.5746] [INSPIRE].

[43] M. Del Zotto, J.J. Heckman, A. Tomasiello and C. Vafa, 6D conformal matter, JHEP 02 (2015) 054 [arXiv: 1407.6359] [INSPIRE].

[44] M. Del Zotto, J.J. Heckman, D.R. Morrison and D.S. Park, 6D SCFTs and gravity, JHEP 06 (2015) 158 [arXiv: 1412.6526] [INSPIRE].

[45] J.J. Heckman, D.R. Morrison, T. Rudelius and C. Vafa, Atomic classification of $6 D$ SCFTs, Fortsch. Phys. 63 (2015) 468 [arXiv:1502.05405] [INSPIRE].

[46] G. Aldazabal, A. Font, L.E. Ibáñez and A.M. Uranga, New branches of string compactifications and their F-theory duals, Nucl. Phys. B 492 (1997) 119 [hep-th/9607121] [INSPIRE].

[47] A. Klemm, P. Mayr and C. Vafa, BPS states of exceptional noncritical strings, hep-th/9607139 [INSPIRE].

[48] A. Klemm, M. Kreuzer, E. Riegler and E. Scheidegger, Topological string amplitudes, complete intersection Calabi-Yau spaces and threshold corrections, JHEP 05 (2005) 023 [hep-th/0410018] [INSPIRE].

[49] T.W. Grimm and T. Weigand, On abelian gauge symmetries and proton decay in global F-theory GUTs, Phys. Rev. D 82 (2010) 086009 [arXiv:1006.0226] [INSPIRE]. 
[50] S. Krause, C. Mayrhofer and T. Weigand, $G_{4}$ flux, chiral matter and singularity resolution in F-theory compactifications, Nucl. Phys. B 858 (2012) 1 [arXiv:1109.3454] [INSPIRE].

[51] T.W. Grimm and H. Hayashi, F-theory fluxes, chirality and Chern-Simons theories, JHEP 03 (2012) 027 [arXiv: 1111.1232] [INSPIRE].

[52] D.S. Park, Anomaly equations and intersection theory, JHEP 01 (2012) 093 [arXiv:1111.2351] [INSPIRE].

[53] M. Esole, J. Fullwood and S.-T. Yau, $D_{5}$ elliptic fibrations: non-Kodaira fibers and new orientifold limits of F-theory, arXiv:1110.6177 [INSPIRE].

[54] M. Cvetič, T.W. Grimm and D. Klevers, Anomaly cancellation and abelian gauge symmetries in F-theory, JHEP 02 (2013) 101 [arXiv:1210.6034] [INSPIRE].

[55] J. Borchmann, C. Mayrhofer, E. Palti and T. Weigand, Elliptic fibrations for $\mathrm{SU}(5) \times \mathrm{U}(1) \times \mathrm{U}(1)$ F-theory vacua, Phys. Rev. D 88 (2013) 046005 [arXiv:1303.5054] [INSPIRE].

[56] M. Cvetič, D. Klevers and H. Piragua, F-theory compactifications with multiple U(1)-factors: constructing elliptic fibrations with rational sections, JHEP 06 (2013) 067 [arXiv:1303.6970] [INSPIRE].

[57] T.W. Grimm, A. Kapfer and J. Keitel, Effective action of 6D F-theory with U(1) factors: rational sections make Chern-Simons terms jump, JHEP 07 (2013) 115 [arXiv:1305.1929] [INSPIRE].

[58] V. Braun, T.W. Grimm and J. Keitel, Geometric engineering in toric F-theory and GUTs with $\mathrm{U}(1)$ gauge factors, JHEP 12 (2013) 069 [arXiv:1306.0577] [INSPIRE].

[59] J. Borchmann, C. Mayrhofer, E. Palti and T. Weigand, SU(5) tops with multiple U(1)s in F-theory, Nucl. Phys. B $\mathbf{8 8 2}$ (2014) 1 [arXiv:1307.2902] [InSPIRE].

[60] M. Cvetič, D. Klevers and H. Piragua, F-theory compactifications with multiple U(1)-factors: addendum, JHEP 12 (2013) 056 [arXiv: 1307.6425] [INSPIRE].

[61] M. Cvetič, D. Klevers, H. Piragua and P. Song, Elliptic fibrations with rank three Mordell-Weil group: F-theory with $\mathrm{U}(1) \times \mathrm{U}(1) \times \mathrm{U}(1)$ gauge symmetry, JHEP 03 (2014) 021 [arXiv: 1310.0463] [INSPIRE].

[62] C. Mayrhofer, D.R. Morrison, O. Till and T. Weigand, Mordell-Weil torsion and the global structure of gauge groups in F-theory, JHEP 10 (2014) 16 [arXiv:1405.3656] [INSPIRE].

[63] V. Braun and D.R. Morrison, F-theory on genus-one fibrations, JHEP 08 (2014) 132 [arXiv: 1401.7844] [INSPIRE].

[64] D.R. Morrison and W. Taylor, Sections, multisections and U(1) fields in F-theory, arXiv: 1404.1527 [INSPIRE].

[65] L.B. Anderson, I. García-Etxebarria, T.W. Grimm and J. Keitel, Physics of F-theory compactifications without section, JHEP 12 (2014) 156 [arXiv:1406.5180] [INSPIRE].

[66] C. Mayrhofer, E. Palti, O. Till and T. Weigand, Discrete gauge symmetries by higgsing in four-dimensional F-theory compactifications, JHEP 12 (2014) 068 [arXiv:1408.6831] [INSPIRE].

[67] I. García-Etxebarria, T.W. Grimm and J. Keitel, Yukawas and discrete symmetries in F-theory compactifications without section, JHEP 11 (2014) 125 [arXiv:1408.6448] [INSPIRE]. 
[68] C. Mayrhofer, E. Palti, O. Till and T. Weigand, On discrete symmetries and torsion homology in F-theory, JHEP 06 (2015) 029 [arXiv:1410.7814] [INSPIRE].

[69] M. Cvetič, R. Donagi, D. Klevers, H. Piragua and M. Poretschkin, F-theory vacua with $Z_{3}$ gauge symmetry, Nucl. Phys. B 898 (2015) 736 [arXiv:1502.06953] [INSPIRE].

[70] M. Kreuzer and H. Skarke, On the classification of reflexive polyhedra, Commun. Math. Phys. 185 (1997) 495 [hep-th/9512204] [INSPIRE].

[71] A. Grassi and V. Perduca, Weierstrass models of elliptic toric K3 hypersurfaces and symplectic cuts, Adv. Theor. Math. Phys. 17 (2013) 741 [arXiv:1201.0930] [InSPIRE].

[72] P. Candelas and A. Font, Duality between the webs of heterotic and type-II vacua, Nucl. Phys. B 511 (1998) 295 [hep-th/9603170] [INSPIRE].

[73] P. Candelas, E. Perevalov and G. Rajesh, Toric geometry and enhanced gauge symmetry of F-theory/heterotic vacua, Nucl. Phys. B 507 (1997) 445 [hep-th/9704097] [InSPIRE].

[74] V. Bouchard and H. Skarke, Affine Kac-Moody algebras, CHL strings and the classification of tops, Adv. Theor. Math. Phys. 7 (2003) 205 [hep-th/0303218] [INSPIRE].

[75] P. Mayr, Mirror symmetry, $N=1$ superpotentials and tensionless strings on Calabi-Yau four folds, Nucl. Phys. B 494 (1997) 489 [hep-th/9610162] [INSPIRE].

[76] A. Klemm, B. Lian, S.S. Roan and S.-T. Yau, Calabi-Yau fourfolds for M-theory and F-theory compactifications, Nucl. Phys. B 518 (1998) 515 [hep-th/9701023] [INSPIRE].

[77] T.W. Grimm, T.-W. Ha, A. Klemm and D. Klevers, Computing brane and flux superpotentials in F-theory compactifications, JHEP 04 (2010) 015 [arXiv:0909. 2025] [INSPIRE].

[78] B.R. Greene, D.R. Morrison and M.R. Plesser, Mirror manifolds in higher dimension, Commun. Math. Phys. 173 (1995) 559 [hep-th/9402119] [INSPIRE].

[79] E. Witten, Nonperturbative superpotentials in string theory, Nucl. Phys. B 474 (1996) 343 [hep-th/9604030] [INSPIRE].

[80] T.W. Grimm, The $N=1$ effective action of F-theory compactifications, Nucl. Phys. B $\mathbf{8 4 5}$ (2011) 48 [arXiv: 1008.4133] [INSPIRE].

[81] E. Witten, On flux quantization in M-theory and the effective action, J. Geom. Phys. 22 (1997) 1 [hep-th/9609122] [INSPIRE].

[82] S. Sethi, C. Vafa and E. Witten, Constraints on low dimensional string compactifications, Nucl. Phys. B 480 (1996) 213 [hep-th/9606122] [INSPIRE].

[83] S. Gukov, C. Vafa and E. Witten, CFT's from Calabi-Yau four folds, Nucl. Phys. B 584 (2000) 69 [Erratum ibid. B 608 (2001) 477] [hep-th/9906070] [INSPIRE].

[84] H. Jockers, P. Mayr and J. Walcher, On $N=14 D$ effective couplings for F-theory and heterotic vacua, Adv. Theor. Math. Phys. 14 (2010) 1433 [arXiv:0912.3265] [INSPIRE].

[85] K. Intriligator, H. Jockers, P. Mayr, D.R. Morrison and M.R. Plesser, Conifold transitions in M-theory on Calabi-Yau Fourfolds with background fluxes, Adv. Theor. Math. Phys. 17 (2013) 601 [arXiv: 1203.6662] [INSPIRE].

[86] N.C. Bizet, A. Klemm and D.V. Lopes, Landscaping with fluxes and the E8 Yukawa point in F-theory, arXiv:1404.7645 [INSPIRE]. 
[87] M. Haack and J. Louis, $M$ theory compactified on Calabi-Yau fourfolds with background flux, Phys. Lett. B 507 (2001) 296 [hep-th/0103068] [INSPIRE].

[88] D. Belov and G.W. Moore, Classification of abelian spin chern-Simons theories, hep-th/0505235 [INSPIRE].

[89] A. Kapustin and N. Saulina, Topological boundary conditions in abelian Chern-Simons theory, Nucl. Phys. B 845 (2011) 393 [arXiv:1008.0654] [INSPIRE].

[90] T.W. Grimm and R. Savelli, Gravitational instantons and fluxes from $M / F$-theory on Calabi-Yau fourfolds, Phys. Rev. D 85 (2012) 026003 [arXiv:1109.3191] [INSPIRE].

[91] A.J. Niemi and G.W. Semenoff, Axial anomaly induced fermion fractionization and effective gauge theory actions in odd dimensional space-times, Phys. Rev. Lett. 51 (1983) 2077 [INSPIRE].

[92] A.N. Redlich, Parity violation and gauge noninvariance of the effective gauge field action in three-dimensions, Phys. Rev. D 29 (1984) 2366 [InSPIRE].

[93] O. Aharony, A. Hanany, K.A. Intriligator, N. Seiberg and M.J. Strassler, Aspects of $N=2$ supersymmetric gauge theories in three-dimensions, Nucl. Phys. B 499 (1997) 67 [hep-th/9703110] [INSPIRE].

[94] T.W. Grimm, D. Klevers and M. Poretschkin, Fluxes and warping for gauge couplings in F-theory, JHEP 01 (2013) 023 [arXiv: 1202.0285] [INSPIRE].

[95] T.W. Grimm and A. Kapfer, Anomaly cancelation in field theory and F-theory on a circle, arXiv:1502.05398 [INSPIRE].

[96] F. Bonetti and T.W. Grimm, Six-dimensional $(1,0)$ effective action of F-theory via M-theory on Calabi-Yau threefolds, JHEP 05 (2012) 019 [arXiv:1112.1082] [INSPIRE].

[97] M.B. Green and J.H. Schwarz, Anomaly cancellation in supersymmetric $D=10$ gauge theory and superstring theory, Phys. Lett. B 149 (1984) 117 [INSPIRE].

[98] A. Sagnotti, A note on the Green-Schwarz mechanism in open string theories, Phys. Lett. B 294 (1992) 196 [hep-th/9210127] [rNSPIRE].

[99] E. Witten, An SU(2) anomaly, Phys. Lett. B 117 (1982) 324 [inSPIRE].

[100] M. Bies, C. Mayrhofer, C. Pehle and T. Weigand, Chow groups, Deligne cohomology and massless matter in F-theory, arXiv: 1402.5144 [INSPIRE].

[101] A. Font and L.E. Ibáñez, Yukawa structure from U(1) fluxes in F-theory grand unification, JHEP 02 (2009) 016 [arXiv:0811.2157] [INSPIRE].

[102] J.J. Heckman and C. Vafa, Flavor hierarchy from F-theory, Nucl. Phys. B 837 (2010) 137 [arXiv:0811.2417] [INSPIRE].

[103] A. Font, L.E. Ibáñez, F. Marchesano and D. Regalado, Non-perturbative effects and Yukawa hierarchies in F-theory SU(5) unification, JHEP 03 (2013) 140 [Erratum ibid. 1307 (2013) 036] [arXiv: 1211.6529] [INSPIRE].

[104] R. Barbier et al., R-parity violating supersymmetry, Phys. Rept. 420 (2005) 1 [hep-ph/0406039] [INSPIRE].

[105] I. Antoniadis and G.K. Leontaris, A supersymmetric $\mathrm{SU}(4) \times O(4)$ model, Phys. Lett. B 216 (1989) 333 [INSPIRE]. 
[106] S.F. King and Q. Shafi, Minimal supersymmetric $\mathrm{SU}(4) \times \mathrm{SU}(2) L \times \mathrm{SU}(2) R$, Phys. Lett. B 422 (1998) 135 [hep-ph/9711288] [INSPIRE].

[107] G. Lazarides, C. Panagiotakopoulos and Q. Shafi, Supersymmetric unification without proton decay, Phys. Lett. B 315 (1993) 325 [Erratum ibid. B 317 (1993) 661] [hep-ph/9306332] [INSPIRE].

[108] G.R. Dvali and Q. Shafi, Gauge hierarchy in $\mathrm{SU}(3)(C) \times \mathrm{SU}(3)(L) \times \mathrm{SU}(3) R$ and low-energy implications, Phys. Lett. B 326 (1994) 258 [hep-ph/9401337] [InSPIRE].

[109] G. Dvali and Q. Shafi, Supersymmetric trinification and low energy consequences, in the proceedings of 1996 Summer school in High Energy Physics and Cosmology, E. Gava et al. eds., World Scientific, Singapore (1996).

[110] T.W. Grimm, Axion inflation in F-theory, Phys. Lett. B 739 (2014) 201 [arXiv: 1404.4268] [INSPIRE].

[111] I. García-Etxebarria, T.W. Grimm and I. Valenzuela, Special points of inflation in flux compactifications, arXiv:1412.5537 [INSPIRE]. 